\title{
THE APPROACH TO NORMALITY OF THE SOLUTIONS OF RANDOM BOUNDARY AND EIGENVALUE PROBLEMS WITH WEAKLY CORRELATED COEFFICIENTS*
}

\author{
BY \\ WILLIAM E. BOYCE (Rensselaer Polytechnic Institute) \\ AND \\ NING-MAO XIA (East China Institute of Chemical Technology)
}

\begin{abstract}
A general class of linear self-adjoint random boundary value problems with weakly correlated coefficients is considered. The earlier result that the distribution function of the solution approaches the normal as the correlation length $\varepsilon$ tends to zero is generalized somewhat. Correction terms are derived that yield estimates for the distribution function when $\varepsilon$ is small but nonzero. The results are also applied to the eigenvalues and eigenfunctions of a corresponding class of random eigenvalue problems. The discussion is given in terms of second-order equations, but extensions to higher-order problems are readily apparent.
\end{abstract}

1. Introduction. For many years it has been of interest to find conditions under which the distribution of the solution of a random differential equation tends to the normal. In 1930, while studying Brownian motion, Uhlenbeck and Ornstein [8] established that the solution $y(t)$ of certain initial-value problems has approximately a normal distribution. They accomplished this by showing that

$$
\begin{aligned}
\left\langle y^{n}\right\rangle & \cong 0, & & n \text { odd } \\
& \cong 1 \cdot 3 \cdot 5 \cdots(n-1)\left\langle y^{2}\right\rangle^{n / 2}, & & n \text { even }
\end{aligned}
$$

where $\langle\cdot\rangle$ denotes the mathematical expectation.

In 1966 Boyce [1] established a similar result for a class of linear self-adjoint boundary value problems

$$
L[y]=f(x), \quad 0<x<1
$$

with boundary conditions

$$
U_{i}[y]=0
$$

* Received October 9, 1981; revised version received January 18, 1982. The results presented in this paper were obtained in the course of research sponsored in part by the National Science Foundation under Grant MCS-8003035. The work was carried out while the second author was a visiting scholar at Rensselaer Polytechnic Institute. 
at the end points. The operator $L$ has the form

$$
L[y]=\sum_{j=0}^{m}(-1)^{j}\left[r_{j}(x) y^{(j)}\right]^{(j)} .
$$

Randomness entered the problem (1.2), (1.3) only through the forcing function $f$, which was assumed to be weakly correlated with correlation length $\varepsilon \ll 1$. The solution $y(x)$ was shown to satisfy equations similar to (1.1), with the approximation becoming exact as $\varepsilon \rightarrow 0$. This means that the distribution of $y(x) / \sqrt{ } \varepsilon$ approaches a normal distribution as $\varepsilon \rightarrow 0$. Combining the methods of [7] and [1] with a perturbation expansion, Purkert and vom Scheidt $[4,7]$ generalized this result to the case in which $f, r_{0}, \ldots, r_{m-1}$ are independent and weakly correlated processes. In Sec. 2 we extend this result still further so as to permit $r_{m}$ also to be a weakly correlated stochastic process.

In many problems arising in applications the correlation length $\varepsilon$ is small, but not vanishingly so. This raises the question of how far the solution $y(x)$ departs from normality when $\varepsilon$ is a small positive number. To answer this question we calculate the second nonzero term in the Hermite-Chebyshev expansion of the distribution function of $y(x)$; this is the most important contribution of the present paper. It turns out that two cases must be considered, depending on whether the correction term is of order $\sqrt{ } \varepsilon$ or of order $\varepsilon$, and these are discussed in Secs. 3 and 4 respectively. Sec. 5 is devoted to an example that gives some feeling for the orders of magnitude of the terms. Purkert and vom Scheidt $[3,5,6,7]$ have also considered self-adjoint eigenvalue problems

$$
\begin{gathered}
L[u]=\lambda u, \quad 0<x<1, \\
U_{i}[u]=0,
\end{gathered}
$$

where the coefficients in $L$ are weakly correlated processes with common correlation length $\varepsilon$. They showed in [5] that $\left(\lambda_{j}-\mu_{j}\right) / \sqrt{ } \varepsilon$ approaches a normal random variable as $\varepsilon \rightarrow 0$. Here $\mu_{j}$ is the $j$ th eigenvalue of the mean problem obtained by replacing each random coefficient in $L$ by its mean. At the same time they established the asymptotic normality of the eigenfunctions of (1.5), (1.6). By proceeding much as in Secs. 3 and 4 it is possible to determine correction terms for the distributions of the eigenvalues and eigenfunctions for small positive values of $\varepsilon$. These matters are discussed briefly in Sec. 6 .

In [3] and [5], in particular, Purkert and vom Scheidt have also developed much necessary background material in a detailed and formal manner. We will use their definitions and nomenclature in this paper without explicit reference.

2. Boundary-value problems with weakly correlated coefficients. Although the same methods can be applied to higher-order problems, in this section we consider the nonhomogeneous two-point boundary-value problem consisting of the second-order differential equation

$$
L[y]=-\left[p(x, \omega) y^{\prime}\right]^{\prime}+q(x, \omega) y=f(x, \omega), \quad 0<x<1,
$$

and the boundary conditions

$$
U_{i}[y]=0 ; \quad i=1,2 .
$$

The coefficients in the differential equation (2.1) are stochastic processes defined on an underlying probability space $(\Omega, \mathscr{F}, P)$. Except possibly on an $\omega$-set of probability zero, the sample functions of $p, q$, and $f$ satisfy the standard conditions: $f$ and $q$ are continuous, 
$p$ is continuously differentiable, and $p$ is never zero in $0 \leq x \leq 1$. The emphasis here will be on the consequences of assuming that the leading coefficient $p(x, \omega)$ is random, since this extends the results of [4]. This section also provides a framework for the following sections.

The boundary conditions (2.2) are deterministic and are such that the problem is self-adjoint. For example, for the problem (2.1), (2.2) it is sufficient that the boundary conditions be separated, in which case

$$
\begin{aligned}
& U_{1}[y]=a_{1} y(0)+a_{2} y^{\prime}(0)=0, \\
& U_{2}[y]=b_{1} y(1)+b_{2} y^{\prime}(1)=0,
\end{aligned}
$$

where $a_{1}, a_{2}, b_{1}$, and $b_{2}$ are given constants.

We can express $p, q$, and $f$ as a sum of their respective means and a random fluctuation:

$$
\begin{array}{ll}
p(x, \omega)=p_{0}(x)+\eta p_{1}(x, \omega), & p_{0}(x)=\langle p(x, \omega)\rangle, \\
q(x, \omega)=q_{0}(x)+\eta q_{1}(x, \omega), & q_{0}(x)=\langle q(x, \omega)\rangle, \\
f(x, \omega)=f_{0}(x)+\eta f_{1}(x, \omega), & f_{0}(x)=\langle f(x, \omega)\rangle,
\end{array}
$$

where

$$
\left\langle p_{1}(x, \omega)\right\rangle=\left\langle q_{1}(x, \omega)\right\rangle=\left\langle f_{1}(x, \omega)\right\rangle=0
$$

and $\eta$ is an indexing parameter; sometimes $\eta=1$. In addition, we assume that $p_{1}, q_{1}$, and $f_{1}$ are pairwise independent wide-sense stationary processes.

We are interested in the case in which $p_{1}, q_{1}$, and $f_{1}$ are also weakly correlated, a term that has been defined by Purkert and vom Scheidt $[4,5]$ in the following way. Let $S=$ $\left(x_{1}, \ldots, x_{n}\right)$ be an $n$-tuple of real numbers and let $\varepsilon>0$ be a positive constant. Let $S_{1}=$ $\left(x_{i_{1}}, \ldots, x_{i_{k}}\right)$ be a subset of $S$, and suppose that $x_{i_{1}} \leq x_{i_{2}} \leq \cdots \leq x_{i_{k}}$; this ordering can always be attained by relabeling the elements of $S_{1}$ if necessary. Then $S_{1}$ is said to be $\varepsilon$-neighboring if

$$
\left|x_{i_{1}}-x_{i_{2}}\right| \leq \varepsilon, \quad\left|x_{i_{2}}-x_{i_{3}}\right| \leq \varepsilon, \ldots, \quad\left|x_{i_{k-1}}-x_{i_{k}}\right| \leq \varepsilon .
$$

A single element subset is always $\varepsilon$-neighboring. The subset $S_{1}$ is maximally $\varepsilon$ neighboring, with respect to $S$, if $S_{1}$ is $\varepsilon$-neighboring, but is not contained in any larger $\varepsilon$-neighboring subset of $S$. It can be shown [5] that $S$ can be separated into disjoint maximally $\varepsilon$-neighboring subsets in a unique way. Then a stationary process $h(x, \omega)$ is said to be weakly correlated with correlation length $\varepsilon$ if, for each $n$,

$$
\left\langle h\left(x_{1}, \omega\right) \cdots h\left(x_{n}, \omega\right)\right\rangle=\left\langle h\left(x_{11}\right) \cdots h\left(x_{1 p_{1}}\right)\right\rangle \cdots\left\langle h\left(x_{k 1}\right) \cdots h\left(x_{k p_{k}}\right)\right\rangle,
$$

where the $n$-tuple $S$ has been separated into the maximally $\varepsilon$-neighboring subsets $\left(x_{11}, \ldots\right.$, $\left.x_{1 p_{1}}\right), \ldots,\left(x_{k 1}, \ldots x_{k p_{k}}\right)$ and $\sum_{i=1}^{k} p_{i}=n$.

Thus we assume that $p_{1}, q_{1}$, and $f_{1}$ have the property (2.6). In the particular case where $n=2$ this reduces to

$$
\begin{aligned}
\left\langle p_{1}\left(x_{1}\right) p_{1}\left(x_{2}\right)\right\rangle & =0, & & \left|x_{2}-x_{1}\right|>\varepsilon ; \\
& =\sigma_{p}^{2} \rho_{p}\left(x_{2}-x_{1}\right), & & \left|x_{2}-x_{1}\right| \leq \varepsilon ;
\end{aligned}
$$




$$
\begin{aligned}
\left\langle q_{1}\left(x_{1}\right) q_{1}\left(x_{2}\right)\right\rangle & =0, & & \left|x_{2}-x_{1}\right|>\varepsilon ; \\
& =\sigma_{q}^{2} \rho_{q}\left(x_{2}-x_{1}\right), & & \left|x_{2}-x_{1}\right| \leq \varepsilon ; \\
\left\langle f_{1}\left(x_{1}\right) f_{1}\left(x_{2}\right)\right\rangle & =0, & & \left|x_{2}-x_{1}\right|>\varepsilon ; \\
& =\sigma_{f}^{2} \rho_{f}\left(x_{2}-x_{1}\right), & & \left|x_{2}-x_{1}\right| \leq \varepsilon .
\end{aligned}
$$

In (2.7) through (2.9) $\sigma_{p}^{2}, \sigma_{q}^{2}$, and $\sigma_{f}^{2}$ are the (constant) variances and $\rho_{p}, \rho_{q}$, and $\rho_{f}$ are the autocorrelation functions of $p_{1}, q_{1}$, and $f_{1}$ respectively. Eq. (2.7) says that $p_{1}\left(x_{1}\right)$ and $p_{1}\left(x_{2}\right)$ are uncorrelated except in a strip of width $\sqrt{ } 2 \varepsilon$ about the line $x_{1}=x_{2}$, and similarly for (2.8) and (2.9).

Finally, we assume that the distributions of $p_{1}, q_{1}$, and $f_{1}$ are such as to allow all of the analytical procedures that we will use without specific justification. This includes the interchange of the order of various types of limiting processes.

It is convenient to write $y(x, \omega)$ as a perturbation series in $\eta$,

$$
y(x, \omega)=y_{0}(x)+\sum_{k=1}^{\infty} y_{k}(x, \omega) \eta^{k} .
$$

Substituting this expression for $y$ in (2.1) and (2.2) and equating coefficients of like powers of $\eta$, we find that $y_{0}(x)$ satisfies

$$
\begin{gathered}
L_{0}\left[y_{0}\right] \equiv-\left[p_{0}(x) y_{0}^{\prime}\right]^{\prime}+q_{0}(x) y_{0}=f_{0}(x), \quad 0<x<1, \\
U_{i}\left[y_{0}\right]=0 ; \quad i=1,2 .
\end{gathered}
$$

Further, from the linear terms in $\eta$, we have

$$
\begin{gathered}
L_{0}\left[y_{1}\right]=f_{1}(x, \omega)-q_{1}(x, \omega) y_{0}(x)+\left[p_{1}(x, \omega) y_{0}^{\prime}(x)\right]^{\prime}, \quad 0<x<1 \\
U_{i}\left[y_{1}\right]=0 ; \quad i=1,2 .
\end{gathered}
$$

In general, for $k \geq 2$ :

$$
\begin{gathered}
L_{0}\left[y_{k}\right]=-q_{1}(x, \omega) y_{k-1}(x, \omega)+\left[p_{1}(x, \omega) y_{k-1}^{\prime}(x, \omega)\right]^{\prime}, \quad 0<x<1 \\
U_{i}\left[y_{k}\right]=0 ; \quad i=1,2 .
\end{gathered}
$$

If zero is not an eigenvalue of $L_{0}$ subject to the boundary conditions (2.12), then $y_{0}(x)$ is given by

$$
y_{0}(x)=\int_{0}^{1} G\left(x, x_{1}\right) f_{0}\left(x_{1}\right) d x_{1},
$$

where $G\left(x, x_{1}\right)$ is the (deterministic) Green's function associated with $L_{0}$ and the given boundary conditions. In the same way

$$
y_{1}(x, \omega)=\int_{0}^{1} G\left(x, x_{1}\right) g_{1}\left(x_{1}, \omega\right) d x_{1}
$$

where $g_{1}$ is the expression on the right-hand side of (2.13):

$$
g_{1}(x, \omega)=f_{1}(x, \omega)-q_{1}(x, \omega) y_{0}(x)+\left[p_{1}(x, \omega) y_{0}^{\prime}(x)\right]^{\prime} .
$$

Similar formulas can be written down for $y_{2}(x, \omega), y_{3}(x, \omega), \ldots$ 
Our primary interest is the calculation of moments of $y_{1}(x, \omega)$. These are given by the expressions

$$
\begin{gathered}
\left\langle y_{1}(x, \omega)\right\rangle=\int_{0}^{1} G\left(x, x_{1}\right)\left\langle g_{1}\left(x_{1}, \omega\right)\right\rangle d x_{1}, \\
\left\langle y_{1}^{2}(x, \omega)\right\rangle=\int_{0}^{1} \int_{0}^{1} G\left(x, x_{1}\right) G\left(x, x_{2}\right)\left\langle g_{1}\left(x_{1}, \omega\right) g_{1}\left(x_{2}, \omega\right)\right\rangle d x_{1} d x_{2},
\end{gathered}
$$

and in general by

$$
\left\langle y_{1}^{n}(x, \omega)\right\rangle=\int_{0}^{1} \cdots \int_{0}^{1} G\left(x, x_{1}\right) \cdots G\left(x, x_{n}\right)\left\langle g_{1}\left(x_{1}, \omega\right) \cdots g_{1}\left(x_{n}, \omega\right)\right\rangle d x_{1} \cdots d x_{n} .
$$

To evaluate these expressions it is necessary to consider some properties of $g_{1}(x, \omega)$. Hereafter, for the sake of brevity, we will usually omit explicit indication of $\omega$ as an independent variable.

In the first place, we have

$$
\begin{aligned}
\left\langle p_{1}^{\prime}(x)\right\rangle & =\left\langle\lim _{\Delta \rightarrow 0} \frac{p_{1}(x+\Delta)-p_{1}(x)}{\Delta}\right\rangle \\
& =\lim _{\Delta \rightarrow 0} \frac{1}{\Delta}\left\langle p_{1}(x+\Delta)-p_{1}(x)\right\rangle=0
\end{aligned}
$$

because of (2.5). Then

$$
\begin{aligned}
\left\langle g_{1}(x)\right\rangle & =\left\langle f_{1}(x)-q_{1}(x) y_{0}(x)+p_{1}^{\prime}(x) y_{0}^{\prime}(x)+p_{1}(x) y_{0}^{\prime \prime}(x)\right\rangle \\
& =\left\langle f_{1}(x)\right\rangle-\left\langle q_{1}(x)\right\rangle y_{0}(x)+\left\langle p_{1}^{\prime}(x)\right\rangle y_{0}^{\prime}(x)+\left\langle p_{1}(x)\right\rangle y_{0}^{\prime \prime}(x) \\
& =0
\end{aligned}
$$

by (2.5) and (2.23). Consequently, from (2.20),

$$
\left\langle y_{1}(x, \omega)\right\rangle=I_{1}(x)=0 .
$$

Using (2.19) to form the product $g_{1}\left(x_{1}\right) g_{1}\left(x_{2}\right)$ and then taking the mean, we obtain

$$
\begin{aligned}
\left\langle g_{1}\left(x_{1}\right) g_{1}\left(x_{2}\right)\right\rangle=\left\langle f_{1}\left(x_{1}\right) f_{1}\left(x_{2}\right)\right\rangle+\left\langle q_{1}\left(x_{1}\right) q_{1}\left(x_{2}\right)\right\rangle & y_{0}\left(x_{1}\right) y_{0}\left(x_{2}\right) \\
& +\left\langle\left[p_{1}\left(x_{1}\right) y_{0}^{\prime}\left(x_{1}\right)\right]^{\prime}\left[p_{1}\left(x_{2}\right) y_{0}^{\prime}\left(x_{2}\right)\right]^{\prime}\right\rangle ;
\end{aligned}
$$

we have used (2.5) and the independence of $f_{1}, p_{1}$, and $q_{1}$ to eliminate cross-product terms such as $\left\langle f_{1}\left(x_{1}\right) q_{1}\left(x_{2}\right)\right\rangle y_{0}\left(x_{2}\right)$. The last term in (2.26) involves the quantities $\left\langle p_{1}\left(x_{1}\right) p_{1}\left(x_{2}\right)\right\rangle$, $\left\langle p_{1}\left(x_{1}\right) p_{1}^{\prime}\left(x_{2}\right)\right\rangle,\left\langle p_{1}^{\prime}\left(x_{1}\right) p_{1}\left(x_{2}\right)\right\rangle$, and $\left\langle p_{1}^{\prime}\left(x_{1}\right) p_{1}^{\prime}\left(x_{2}\right)\right\rangle$. The first of these is given by (2.7), so let us consider the others. We will need to assume that the correlation function $\rho_{p}$ is twice differentiable. First we have

$$
\begin{aligned}
\left\langle p_{1}\left(x_{1}\right) p_{1}^{\prime}\left(x_{2}\right)\right\rangle & =\left\langle p_{1}\left(x_{1}\right) \lim _{\Delta \rightarrow 0} \frac{p_{1}\left(x_{2}+\Delta\right)-p_{1}\left(x_{2}\right)}{\Delta}\right\rangle \\
& =\lim _{\Delta \rightarrow 0} \frac{1}{\Delta}\left[\left\langle p_{1}\left(x_{1}\right) p_{1}\left(x_{2}+\Delta\right)\right\rangle-\left\langle p_{1}\left(x_{1}\right) p_{1}\left(x_{2}\right)\right\rangle\right] .
\end{aligned}
$$


If $\left|x_{2}-x_{1}\right| \leq \varepsilon$ and if $\Delta$ is such that $\left|x_{2}-x_{1}+\Delta\right| \leq \varepsilon$ also, then from (2.7) we have

$$
\begin{aligned}
\left\langle p_{1}\left(x_{1}\right) p_{1}^{\prime}\left(x_{2}\right)\right\rangle & =\sigma_{p}^{2} \lim _{\Delta \rightarrow 0} \frac{1}{\Delta}\left[\rho_{p}\left(x_{2}-x_{1}+\Delta\right)-\rho_{p}\left(x_{2}-x_{1}\right)\right] \\
& =\sigma_{p}^{2} \rho_{p}^{\prime}\left(x_{2}-x_{1}\right), \quad\left|x_{2}-x_{1}\right| \leq \varepsilon .
\end{aligned}
$$

At the endpoints $\rho_{p}^{\prime}$ is interpreted as a one-sided derivative. For $\left|x_{2}-x_{1}\right|>\varepsilon$ and for $\Delta$ small enough so that $\left|x_{2}-x_{1}+\Delta\right|>\varepsilon$ also, then (2.27) and (2.7) yield

$$
\left\langle p_{1}\left(x_{1}\right) p_{1}^{\prime}\left(x_{2}\right)\right\rangle=0, \quad\left|x_{2}-x_{1}\right|>\varepsilon .
$$

In the same way we find that

$$
\begin{aligned}
\left\langle p_{1}^{\prime}\left(x_{1}\right) p_{1}\left(x_{2}\right)\right\rangle & =0, & & \left|x_{2}-x_{1}\right|>\varepsilon ; \\
& =-\sigma_{p}^{2} \rho_{p}^{\prime}\left(x_{2}-x_{1}\right), & & \left|x_{2}-x_{1}\right| \leq \varepsilon .
\end{aligned}
$$

Proceeding analogously with the remaining term, we obtain

$$
\begin{aligned}
\left\langle p_{1}^{\prime}\left(x_{1}\right) p_{1}^{\prime}\left(x_{2}\right)\right\rangle & =0, & & \left|x_{2}-x_{1}\right|>\varepsilon ; \\
& =-\sigma_{p}^{2} \rho_{p}^{\prime \prime}\left(x_{2}-x_{1}\right), & & \left|x_{2}-x_{1}\right| \leq \varepsilon .
\end{aligned}
$$

Finally, substituting from (2.7), (2.8), (2.9), (2.28), (2.29), and (2.30) into (2.26), we have

$$
\begin{aligned}
\left\langle g_{1}\left(x_{1}\right) g_{1}\left(x_{2}\right)\right\rangle= & \sigma_{f}^{2} \rho_{f}\left(x_{2}-x_{1}\right)+\sigma_{q}^{2} \rho_{q}\left(x_{2}-x_{1}\right) y_{0}\left(x_{1}\right) y_{0}\left(x_{2}\right) \\
& +\sigma_{p}^{2} \rho_{p}\left(x_{2}-x_{1}\right) y_{0}^{\prime \prime}\left(x_{1}\right) y_{0}^{\prime \prime}\left(x_{2}\right)-\sigma_{p}^{2} \rho_{p}^{\prime \prime}\left(x_{2}-x_{1}\right) y_{0}^{\prime}\left(x_{1}\right) y_{0}^{\prime}\left(x_{2}\right) \\
& +\sigma_{p}^{2} \rho_{p}^{\prime}\left(x_{2}-x_{1}\right)\left[y_{0}^{\prime \prime}\left(x_{1}\right) y_{0}^{\prime}\left(x_{2}\right)-y_{0}^{\prime}\left(x_{1}\right) y_{0}^{\prime \prime}\left(x_{2}\right)\right], \quad\left|x_{2}-x_{1}\right| \leq \varepsilon,
\end{aligned}
$$

and

$$
\left\langle g_{1}\left(x_{1}\right) g_{1}\left(x_{2}\right)\right\rangle=0, \quad\left|x_{2}-x_{1}\right|>\varepsilon .
$$

This, in turn, gives $\left\langle y_{1}^{2}(x)\right\rangle$ from (2.21). To simplify the latter expression it is convenient to introduce the new coordinates (Fig. 1)

$$
s=x_{2}-x_{1}, \quad z=\left(x_{1}+x_{2}\right) / 2
$$

or

$$
x_{1}=z-s / 2, \quad x_{2}=z+s / 2 .
$$

Then, since the integrand is even in $s$, we can write

$$
\left\langle y_{1}^{2}(x)\right\rangle=2 \int_{0}^{1} \int_{0}^{\varepsilon} G\left(x, z-\frac{s}{2}\right) G\left(x, z+\frac{s}{2}\right)\left\langle g_{1}\left(z-\frac{s}{2}\right) g_{1}\left(z+\frac{s}{2}\right)\right\rangle d s d z-2 J_{1}-2 J_{2},
$$

where $J_{1}$ and $J_{2}$ are the integrals of the same integrand over the triangular regions $T_{1}$ and $T_{2}$ respectively. Note that $J_{1}$ and $J_{2}$ are of order $\varepsilon^{2}$. By expanding the integrand in powers of $s$ and keeping only the first terms, we obtain the contribution to $\left\langle y_{1}^{2}(x)\right\rangle$ that is linear in $\varepsilon$, namely,

$$
\left\langle y_{1}^{2}(x)\right\rangle=I_{2}(x)=A_{1}(x) \varepsilon+O\left(\varepsilon^{2}\right)
$$




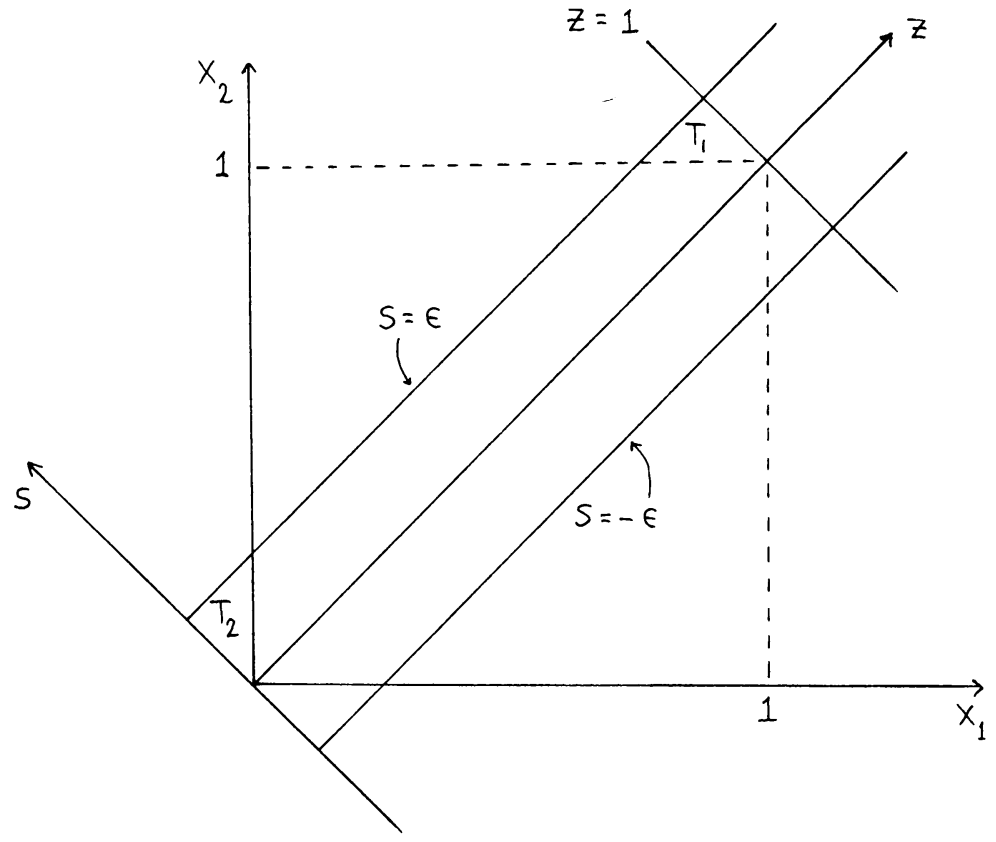

Fig. 1.

where

$$
A_{1}(x)=2 \int_{0}^{1} G^{2}(x, z)\left[\sigma_{f}^{2}+\sigma_{q}^{2} y_{0}^{2}(z)+\sigma_{p}^{2}\left\{y_{0}^{\prime \prime 2}(z)-\rho_{p}^{\prime \prime}(0) y_{0}^{\prime 2}(z)\right\}\right] d z
$$

To evaluate higher moments it is necessary to use (2.22). It is possible, although somewhat tedious, to show that even though $g_{1}(x, \omega)$ is not stationary, it nevertheless has the property (2.6). It is also helpful to define the set $R_{k}$ by

$$
R_{k}=\left\{\left(x_{1}, \ldots, x_{k}\right) \mid 0 \leq x_{1}, \ldots, x_{k} \leq 1 \text { and }\left(x_{1}, \ldots, x_{k}\right) \text { is } \varepsilon \text {-neighboring }\right\} ;
$$

it is clear that the volume of $R_{k}$ is of order $\varepsilon^{k-1}$. Examining (2.22) first for $n=3$, we note that the only contribution to $\left\langle y_{1}^{3}(x)\right\rangle$ comes from points in $R_{3}$; thus

$$
\begin{aligned}
\left\langle y_{1}^{3}(x)\right\rangle & =\iiint_{R_{3}} G\left(x, x_{1}\right) G\left(x, x_{2}\right) G\left(x, x_{3}\right)\left\langle g_{1}\left(x_{1}\right) g_{1}\left(x_{2}\right) g_{1}\left(x_{3}\right)\right\rangle d x_{1} d x_{2} d x_{3} \\
& =I_{3}(x)=A_{3}(x) \varepsilon^{2}+O\left(\varepsilon^{3}\right) .
\end{aligned}
$$

Next, consider $\left\langle y_{1}^{4}(x)\right\rangle$. There is a contribution to this quantity from points in $R_{4}$, and there are other contributions from points where $x_{1}, x_{2}, x_{3}$, and $x_{4}$ are $\varepsilon$-neighboring in pairs. Thus

$$
\begin{aligned}
\left\langle y_{1}^{4}(x)\right\rangle= & 3 I_{2}^{2}(x)+\iiint \int_{R_{4}} G\left(x, x_{1}\right) \cdots G\left(x, x_{4}\right)\left\langle g_{1}\left(x_{1}\right) \cdots g_{1}\left(x_{4}\right)\right\rangle d x_{1} \cdots d x_{4} \\
& -3 \iiint \int_{R_{4}} G\left(x, x_{1}\right) \cdots G\left(x, x_{4}\right)\left\langle g_{1}\left(x_{1}\right) g_{1}\left(x_{2}\right)\right\rangle\left\langle g_{1}\left(x_{3}\right) g_{1}\left(x_{4}\right)\right\rangle d x_{1} \cdots d x_{4} .
\end{aligned}
$$


The coefficient 3 in the first and last terms on the right side of (2.37) arises from the number of ways that four objects can be separated into two parts, while the last term is needed to compensate for the duplication occurring in the first two terms. In the last term, note that the integrand is zero in the part of $R_{4}$ that is not in $R_{2} \times R_{2}$. More briefly, we can rewrite (2.37) as

$$
\left\langle y_{1}^{4}(x)\right\rangle=3 I_{2}^{2}(x)+I_{4}(x),
$$

where $I_{4}(x)$ refers to the last two terms in (2.37), which are of order $\varepsilon^{3}$. The quantity $I_{4}(x)$ will be important later, but for the present we need to keep only the $\varepsilon^{2}$ term. Thus we obtain

$$
\left\langle y_{1}^{4}(x)\right\rangle=3 A_{1}^{2}(x) \varepsilon^{2}+O\left(\varepsilon^{3}\right) .
$$

Similarly,

$$
\left\langle y_{1}^{5}(x)\right\rangle=10 I_{2}(x) I_{3}(x)+O\left(\varepsilon^{4}\right)=10 A_{1}(x) A_{3}(x) \varepsilon^{3}+O\left(\varepsilon^{4}\right) .
$$

In general, we find that

$$
\begin{aligned}
&\left\langle y_{1}^{2 n}(x)\right\rangle=\frac{(2 n) !}{2^{n} n !} I_{2}^{n}(x)+O\left(\varepsilon^{n+1}\right)=\frac{(2 n) !}{2^{n} n !} A_{1}^{n}(x) \varepsilon^{n}+O\left(\varepsilon^{n+1}\right) ; \\
&\left\langle y_{1}^{2 n+1}(x)\right\rangle= \frac{(2 n+1) !}{3 ! 2^{n-1}(n-1) !} I_{2}^{n-1}(x) I_{3}(x)+O\left(\varepsilon^{n+2}\right) \\
&=\frac{(2 n+1) !}{3 ! 2^{n-1}(n-1) !} A_{1}^{n-1}(x) A_{3}(x) \varepsilon^{n+1}+O\left(\varepsilon^{n+2}\right) .
\end{aligned}
$$

We now define the normalized random variable

$$
\xi(x, \omega)=\frac{y_{1}(x, \omega)}{\sqrt{A_{1}(x) \varepsilon}} .
$$

Then

$$
\begin{gathered}
\left\langle\xi^{2 n}\right\rangle=\frac{\left\langle y_{1}^{2 n}\right\rangle}{A_{1}^{n} \varepsilon^{n}}=\frac{(2 n) !}{2^{n} n !}+O(\varepsilon), \\
\left\langle\xi^{2 n+1}\right\rangle=\frac{\left\langle y_{1}^{2 n+1}\right\rangle}{\left(A_{1} \varepsilon\right)^{n+1 / 2}}=\frac{(2 n+1) !}{3 ! 2^{n-1}(n-1) !} \frac{A_{3}}{A_{1}^{3 / 2}} \sqrt{ } \varepsilon+O\left(\varepsilon^{3 / 2}\right) .
\end{gathered}
$$

Thus, as $\varepsilon \rightarrow 0$, the distribution $F_{\xi}$ of $\xi$ approaches the standard normal distribution $\Phi$. In other words,

$$
\lim _{\varepsilon \rightarrow 0} F_{\xi}(u)=\Phi(u)=\frac{1}{\sqrt{2 \pi}} \int_{-\infty}^{u} e^{-t^{2} / 2} d t .
$$

To the extent that the second- and higher-order terms in (2.10) can be neglected, we have shown that the distribution of

$$
\frac{y(x, \omega)-y_{0}(x)}{\sqrt{A_{1}(x) \varepsilon}}
$$

approaches $\Phi$ as $\varepsilon \rightarrow 0$. 
This generalizes the results in [1], [4], and [7] to the case in which the leading coefficient $p$ in (2.1) is random. The further extension to higher-order self-adjoint boundaryvalue problems is straightforward.

3. The distribution function; first correction term. In the preceding section we showed that the distribution function $F_{\xi}$ of the random variable $\xi=y_{1} / \sqrt{A_{1} \varepsilon}$ approaches the standard normal distribution $\Phi$ as the correlation length $\varepsilon \rightarrow 0$. We can look upon this result as generating the first term in an asymptotic expansion of $F_{\xi}$ in terms of $\varepsilon$. In order to investigate more carefully the behavior of $F_{\xi}$ for small but nonzero $\varepsilon$, it is natural to try to determine at least the second term in this expansion. We will do this by means of an expansion in terms of Chebyshev-Hermite polynomials, as outlined by Gnedenko and Kolmogorov [2].

Let $p_{\xi}(u)$ be the density function of $\xi$, and consider the representation

$$
p_{\xi}(u)=\frac{1}{\sqrt{2 \pi}} \sum_{k=0}^{\infty}(-1)^{k} \frac{c_{k}}{k !} e^{-u^{2} / 2} H_{k}(u),
$$

where $H_{k}$ is the Chebyshev-Hermite polynomial of degree $k$. The polynomials $\left\{H_{k}\right\}$ are defined by

$$
H_{k}(u)=(-1)^{k} e^{u^{2} / 2} \frac{d^{k}}{d u^{k}}\left(e^{-u^{2} / 2}\right)
$$

and satisfy the orthogonality relation

$$
\begin{aligned}
\int_{-\infty}^{\infty} e^{-u^{2} / 2} H_{j}(u) H_{k}(u) d u & =0, & & \text { if } j \neq k ; \\
& =\sqrt{2 \pi} k !, & & \text { if } j=k .
\end{aligned}
$$

The first few Chebyshev-Hermite polynomials are

$$
\begin{gathered}
H_{0}(u)=1, \quad H_{1}(u)=u, \quad H_{2}(u)=u^{2}-1, \quad H_{3}(u)=u^{3}-3 u, \\
H^{4}(u)=u^{4}-6 u^{2}+3 .
\end{gathered}
$$

From (3.1) and (3.3) it follows that

$$
c_{k}=(-1)^{k} \int_{-\infty}^{\infty} p_{\xi}(u) H_{k}(u) d u, \quad k=0,1,2, \ldots
$$

In particular,

$$
\begin{gathered}
c_{0}=\int_{-\infty}^{\infty} p_{\xi}(u) d u=1, \\
c_{1}=-\int_{-\infty}^{\infty} u p_{\xi}(u) d u=-\langle\xi\rangle=-\frac{\left\langle y_{1}\right\rangle}{\sqrt{A_{1} \varepsilon}}=0 ;
\end{gathered}
$$

consequently

$$
p_{\xi}(u)=\frac{1}{\sqrt{2 \pi}} e^{-u^{2} / 2}\left[1+\sum_{k=2}^{\infty} \frac{(-1)^{k} c_{k}}{k !} H_{k}(u)\right] .
$$


The corresponding expression for the distribution function $F_{\xi}(u)$ is

$$
F_{\xi}(u)=\Phi(u)+\sum_{k=2}^{\infty} \frac{c_{k}}{k !} \Phi_{k}(u),
$$

where

$$
\Phi_{k}(u)=\frac{(-1)^{k}}{\sqrt{2 \pi}} \int_{-\infty}^{u} e^{-t^{2} / 2} H_{k}(t) d t .
$$

By using (3.2) it follows that

$$
\Phi_{k}(u)=\frac{(-1)^{k-1}}{\sqrt{2 \pi}} e^{-u^{2} / 2} H_{k-1}(u),
$$

so

$$
F_{\xi}(u)=\Phi(u)+\frac{1}{\sqrt{2 \pi}} \sum_{k=2}^{\infty} \frac{(-1)^{k-1} c_{k}}{k !} e^{-u^{2} / 2} H_{k-1}(u) .
$$

Our goal is to determine the contribution to the series (3.11) that is of lowest order in $\varepsilon$. This can be done formally by relating the coefficients $c_{k}$ to the moments of $\xi$, again using some results given in [2].

The characteristic function $\psi_{\xi}(t)$ of $\xi$ is defined by

$$
\psi_{\xi}(t)=\int_{-\infty}^{\infty} e^{i t u} d F_{\xi}(u)
$$

Hence, from (3.8),

$$
\begin{aligned}
\psi_{\xi}(t) & =\int_{-\infty}^{\infty} e^{i t u} d \Phi(u)+\sum_{k=2}^{\infty} \frac{c_{k}}{k !} \int_{-\infty}^{\infty} e^{i t u} d \Phi_{k}(u) \\
& =e^{-t^{2} / 2}\left[1+\sum_{k=2}^{\infty} \frac{c_{k}}{k !}(-i t)^{k}\right]
\end{aligned}
$$

On the other hand, we also have the expansions

$$
\psi_{\xi}(t)=\sum_{k=0}^{\infty} \frac{\alpha_{k}}{k !}(i t)^{k}
$$

where $\alpha_{k}=\left\langle\xi^{k}\right\rangle$, and

$$
\log \psi_{\xi}(t)=\sum_{k=1}^{\omega} \frac{\beta_{k}}{k !}(i t)^{k},
$$

where $\beta_{k}$ is the $k$ th semi-invariant of $\xi$. Starting from the expressions (2.43) and (2.44) for $\alpha_{2 n}$ and $\alpha_{2 n+1}$, we will first determine $\beta_{k}$ and then find $c_{k}$. Throughout this process we will keep terms of order $\sqrt{ } \varepsilon$ and neglect terms of order $\varepsilon$.

To relate $\left\{\beta_{k}\right\}$ with $\left\{\alpha_{k}\right\}$ we need to calculate the logarithm of $\psi_{\xi}(t)$ from (3.14). We first substitute for $\alpha_{k}$ from (2.43) and (2.44), letting $B=A_{3} / A_{1}^{3 / 2}$ and $w=i t$. Then

$$
\begin{aligned}
\log \psi_{\xi}(t) & =\log \sum_{k=0}^{\infty} \frac{\alpha_{k}}{k !} w^{k} \\
& =\log \left[1+\sum_{n=1}^{\infty} \frac{w^{2 n}}{2^{n} n !}+\sum_{n=1}^{\infty} \frac{B \sqrt{ } \varepsilon w^{2 n+1}}{3 ! 2^{n-1}(n-1) !}+O(\varepsilon)\right]
\end{aligned}
$$




$$
\begin{aligned}
& =\log \left[\sum_{n=0}^{\infty} \frac{w^{2 n}}{2^{n} n !}+\frac{B \sqrt{ } \varepsilon w^{3}}{3 !} \sum_{n=1}^{\infty} \frac{w^{2 n-2}}{2^{n-1}(n-1) !}+O(\varepsilon)\right] \\
& =\log \left[\left(1+\frac{B \sqrt{ } \varepsilon w^{3}}{3 !}\right) e^{w^{2} / 2}+O(\varepsilon)\right] \\
& =\frac{w^{2}}{2}+\log \left(1+\frac{B \sqrt{ } \varepsilon w^{3}}{3 !}\right)+O(\varepsilon) \\
& =\frac{w^{2}}{2}+\frac{B \sqrt{ } \varepsilon}{3 !} w^{3}+O(\varepsilon) .
\end{aligned}
$$

Comparing (3.15) with (3.16), and neglecting terms of order $\varepsilon$, we obtain

$$
\beta_{1}=0, \quad \beta_{2}=1, \quad \beta_{3}=B \sqrt{ } \varepsilon, \quad \text { and } \quad \beta_{k}=0 \text { for } k \geq 4 \text {. }
$$

Next we write

$$
\begin{aligned}
\psi_{\xi}(t) & =\exp \left[\log \psi_{\xi}(t)\right]=\exp \left[\sum_{k=1}^{\infty} \frac{\beta_{k}}{k !}(i t)^{k}\right] \\
& =\exp \left[-\frac{t^{2}}{2}+\frac{B \sqrt{ } \varepsilon}{3 !}(i t)^{3}+O(\varepsilon)\right] \\
& =\exp \left(-t^{2} / 2\right) \exp \left[\frac{B \sqrt{ } \varepsilon}{3 !}(i t)^{3}\right]+O(\varepsilon) .
\end{aligned}
$$

By comparing (3.13) with (3.18) we obtain

$$
\begin{aligned}
1+\sum_{k=2}^{\infty} \frac{c_{k}}{k !}(-i t)^{k} & =\exp \left[\frac{B \sqrt{ } \varepsilon}{3 !}(i t)^{3}\right]+O(\varepsilon) \\
& =1+\frac{B \sqrt{ } \varepsilon}{3 !}(i t)^{3}+O(\varepsilon) .
\end{aligned}
$$

Again neglecting terms of order $\varepsilon$, we find that

$$
c_{2}=0, \quad c_{3}=-B \sqrt{ } \varepsilon, \quad \text { and } \quad c_{k}=0 \quad \text { for } \quad k \geq 4 \text {. }
$$

Substituting these coefficients in (3.11) gives the desired result:

$$
\begin{aligned}
F_{\xi}(u) & =\Phi(u)-\frac{1}{\sqrt{2 \pi}} \frac{B \sqrt{ } \varepsilon}{3 !} e^{-u^{2} / 2} H_{2}(u)+O(\varepsilon) \\
& =\frac{1}{\sqrt{2 \pi}} \int_{-\infty}^{u} e^{-t^{2} / 2} d t-\frac{A_{3} \sqrt{ } \varepsilon}{\sqrt{2 \pi} 3 ! A_{1}^{3 / 2}}\left(u^{2}-1\right) e^{-u^{2} / 2}+O(\varepsilon),
\end{aligned}
$$

where $A_{1}$ and $A_{3}$ are defined by (2.35) and (2.36) respectively. The corresponding formula for the density function is

$$
p_{\xi}(u)=\phi(u)\left[1+\frac{A_{3} \sqrt{\varepsilon}}{3 ! A_{1}^{3 / 2}}\left(u^{3}-3 u\right)+O(\varepsilon)\right],
$$


where

$$
\phi(u)=\frac{1}{\sqrt{2 \pi}} e^{-u^{2} / 2}
$$

is the normal density function.

The second term on the right side of (3.21) gives the dominant contribution to $F_{\xi}(u)-\Phi(u)$ when $\varepsilon$ is nonzero but small; at least this is so when $A_{3} \neq 0$. Unfortunately, there are important cases in which $A_{3}=0$. For example, this will occur when the distribution of $\xi$ is symmetric about $\xi=0$. To deal with this situation we must calculate the $O(\varepsilon)$ term in (3.21), and this is done in the next section.

Before turning to that problem, let us briefly consider the calculation of $\left\langle y_{1}^{3}(x)\right\rangle$, which leads to the coefficient $A_{3}$ in (3.21) and (3.22). The only nonzero contribution to the integral

$$
\left\langle y_{1}^{3}(x)\right\rangle=\int_{0}^{1} \int_{0}^{1} \int_{0}^{1} G\left(x, x_{1}\right) G\left(x, x_{2}\right) G\left(x, x_{3}\right)\left\langle g_{1}\left(x_{1}\right) g_{1}\left(x_{2}\right) g_{1}\left(x_{3}\right)\right\rangle d x_{1} d x_{2} d x_{3}
$$

comes from the region where at least one of the following sets of inequalities is satisfied:

$$
\begin{array}{llll} 
& \left|x_{1}-x_{2}\right| \leq \varepsilon & \text { and } & \left|x_{2}-x_{3}\right| \leq \varepsilon ; \\
\text { or } & \left|x_{2}-x_{3}\right| \leq \varepsilon & \text { and } & \left|x_{3}-x_{1}\right| \leq \varepsilon ; \\
\text { or } & \left|x_{3}-x_{1}\right| \leq \varepsilon & \text { and } & \left|x_{1}-x_{2}\right| \leq \varepsilon
\end{array}
$$

This is a right cylinder whose axis is the line $x_{1}=x_{2}=x_{3}$ and whose cross-section in a plane perpendicular to the axis is a regular six-pointed star.

It is helpful to introduce a new orthogonal coordinate system $z, s_{1}, s_{2}$ by the transformation

$$
z=\left(x_{1}+x_{2}+x_{3}\right) / 3, \quad s_{1}=\left(x_{1}-2 x_{2}+x_{3}\right) / \sqrt{ } 2, \quad s_{2}=\left(x_{1}-x_{3}\right) / \sqrt{ } 2 .
$$

The coordinate $z$ lies along the axis of the cylinder, while $s_{1}$ and $s_{2}$ are in a transverse plane. The Jacobian of this transformation is one, and its inverse is

$$
\begin{gathered}
x_{1}=z+\frac{\sqrt{ } 2}{6} s_{1}+\frac{\sqrt{ } 2}{2} s_{2}, \quad x_{2}=z-\frac{\sqrt{ } 2}{3} s_{1}, \\
x_{3}=z+\frac{\sqrt{ } 2}{6} s_{1}-\frac{\sqrt{ } 2}{2} s_{2} .
\end{gathered}
$$

In a plane $z=c$ the cross-section of the cylinder is the star $S$ shown in Fig. 2. The $s_{1}, s_{2}$ coordinates of the points of $S$ are $( \pm 3 \varepsilon / \sqrt{ } 2, \pm \varepsilon / \sqrt{ } 2)$ and $(0, \pm \sqrt{ } 2 \varepsilon)$, where all combinations of plus and minus signs occur. If we introduce the new coordinates in (3.24), expand the integrand in the transverse variables $s_{1}, s_{2}$, and keep only the lowest terms, we find that

$$
\begin{aligned}
\left\langle y_{1}^{3}(x)\right\rangle & =\iint_{S} d s_{1} d s_{2} \int_{0}^{1} G^{3}(x, z)\left\langle g_{1}^{3}(z)\right\rangle d z+O\left(\varepsilon^{3}\right) \\
& =6 \varepsilon^{2} \int_{0}^{1} G^{3}(x, z)\left\langle g_{1}^{3}(z)\right\rangle d z+O\left(\varepsilon^{3}\right),
\end{aligned}
$$




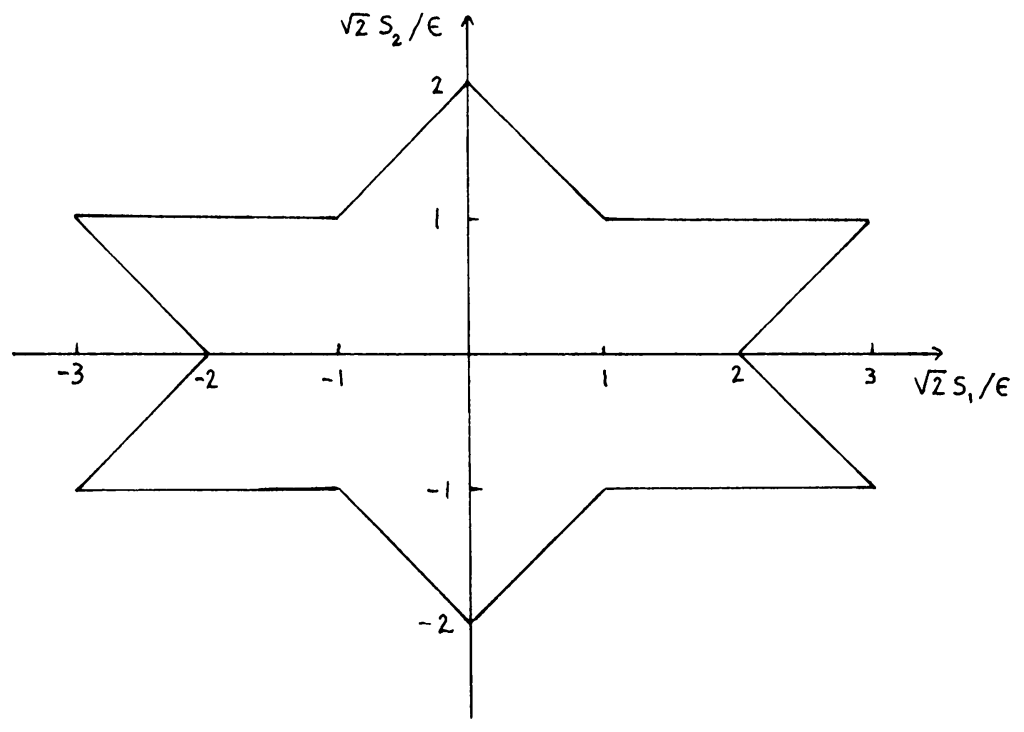

FIG. 2.

where $6 \varepsilon^{2}$ is the area of $S$. The calculation of $\left\langle g_{1}^{3}(z)\right\rangle$ is greatly simplified by the assumption that $p_{1}, q_{1}$, and $f_{1}$ are independent and the fact that $\left\langle p_{1}\right\rangle,\left\langle p_{1}^{\prime}\right\rangle,\left\langle q_{1}\right\rangle$, and $\left\langle f_{1}\right\rangle$ are all zero. Thus most of the terms in $\left\langle g_{1}^{3}(z)\right\rangle$ are zero, and

$$
\left\langle g_{1}^{3}(z)\right\rangle=\left\langle f_{1}^{3}\right\rangle-\left\langle q_{1}^{3}\right\rangle y_{0}^{3}+\left\langle\left\{\left[p_{1} y_{0}^{\prime}\right]^{\prime}\right\}^{3}\right\rangle .
$$

Since $A_{3}$ is the coefficient of $\varepsilon^{2}$ in (3.28), we have

$$
A_{3}(x)=6 \int_{0}^{1} G^{3}(x, z)\left\langle g_{1}^{3}(z)\right\rangle d z,
$$

where $\left\langle g_{1}^{3}(z)\right\rangle$ is given by (3.29). A further determination of $A_{3}$ requires some specific hypotheses about the distributons of $p_{1}, q_{1}$, and $f_{1}$.

4. The distribution function; second correction term. In order to deal with the case in which $A_{3}(x)=0$, and hence $\left\langle y_{1}^{3}(x)\right\rangle=O\left(\varepsilon^{3}\right)$, we must determine the $O(\varepsilon)$ term on the right side of (3.21). In turn, this requires the determination of higher-order terms in $I_{2}(x)$ and also a consideration of $I_{4}(x)$. We write

$$
\begin{aligned}
& I_{2}(x)=A_{1}(x) \varepsilon+A_{2}(x) \varepsilon^{2}+O\left(\varepsilon^{3}\right), \\
& I_{3}(x)=0+O\left(\varepsilon^{3}\right), \\
& I_{4}(x)=A_{4}(x) \varepsilon^{3}+O\left(\varepsilon^{4}\right),
\end{aligned}
$$

where $A_{1}(x)$ is the same as before, $A_{3}(x)$ has been set equal to zero, and the calculation of $A_{2}(x)$ and $A_{4}(x)$ will be discussed later. We will follow the same line of argument as in Sec. 3. We will first determine the moments $\left\langle y_{1}^{k}(x)\right\rangle$ or $\left\langle\xi^{k}(x)\right\rangle$, which give the coefficients $\alpha_{k}$ in the series (3.14) for the characteristic function $\psi_{\xi}(t)$. Next we find the semi-invariants $\beta_{k}$, and finally the coefficients $c_{k}$ in the series (3.11) for $F_{\xi}(u)$. Throughout the derivation we will keep the terms that contribute to order $\varepsilon$ in $F_{\xi}(u)$. 
By keeping the $O\left(\varepsilon^{n+1}\right)$ term in $\left\langle y_{1}^{2 n}\right\rangle$ we obtain, for $n \geq 2$,

$$
\left\langle y_{1}^{2 n}(x)\right\rangle=\frac{(2 n) !}{2^{n} n !} I_{2}^{n}(x)+\frac{(2 n) !}{2^{n-2}(n-2) ! 4 !} I_{2}^{n-2}(x) I_{4}(x)+O\left(\varepsilon^{n+2}\right) .
$$

The first term on the right side was obtained in $(2.40)$ and is $O\left(\varepsilon^{n}\right)$. The second term is the contribution of order $\varepsilon^{n+1}$; its coefficient comes from a consideration of the number of ways that one set of four and $n-2$ pairs can be chosen from a set of $2 n$ objects. Note that if $A_{3}(x)$ were not zero, then there would be another contribution of order $\varepsilon^{n+1}$ of the form $I_{2}^{n-3}(x) I_{3}^{2}(x)$. From (4.1) and the binomial theorem it follows that

$$
I_{2}^{n}=\left(A_{1} \varepsilon\right)^{n}\left[1+n \frac{A_{2}}{A_{1}} \varepsilon\right]+O\left(\varepsilon^{n+2}\right)
$$

Then

$$
\left\langle y_{1}^{2 n}\right\rangle=\frac{(2 n) !}{2^{n} n !}\left(A_{1} \varepsilon\right)^{n}\left[1+n \frac{A_{2}}{A_{1}} \varepsilon\right]+\frac{(2 n) !}{2^{n-2}(n-2) ! 4 !}\left(A_{1} \varepsilon\right)^{n-2} A_{4} \varepsilon^{3}+O\left(\varepsilon^{n+2}\right) .
$$

Since $\xi(x)=y_{1}(x) / \sqrt{A_{1}(x) \varepsilon}$, we have

$$
\begin{aligned}
\alpha_{2 n} & =\left\langle\xi^{2 n}\right\rangle=\left\langle y_{1}^{2 n}\right\rangle /\left(A_{1} \varepsilon\right)^{n} \\
& =(2 n) !\left\{\frac{1}{2^{n} n !}+\left[\frac{A_{2} / A_{1}}{2^{n}(n-1) !}+\frac{A_{4} / A_{1}^{2}}{2^{n-2}(n-2) ! 4 !}\right] \varepsilon\right\}+O\left(\varepsilon^{2}\right), n \geq 2 .
\end{aligned}
$$

Of course,

$$
\alpha_{0}=1, \quad \alpha_{2}=1+\left(A_{2} / A_{1}\right) \varepsilon+O\left(\varepsilon^{2}\right) .
$$

Also, because $A_{3}(x)=0$, we have $\left\langle y_{1}^{2 n+1}(x)\right\rangle=O\left(\varepsilon^{n+2}\right)$, and

$$
\alpha_{2 n+1}=\left\langle\xi^{2 n+1}\right\rangle=O\left(\varepsilon^{3 / 2}\right) \text {. }
$$

Next we consider

$$
\log \psi_{\xi}(t)=\log \left[\sum_{k=0}^{\infty} \frac{\alpha_{k}}{k !} w^{k}\right],
$$

where $w=i t$. Since to order $\varepsilon$ only the even-powered terms contribute, we can write

$$
\begin{aligned}
\log \psi_{\xi}(t)= & \log \left\{1+\left(1+\frac{A_{2}}{A_{1}} \varepsilon\right) \frac{w^{2}}{2}+\sum_{n=2}^{\infty}\left[\frac{1}{2^{n} n !}+\varepsilon\left(\frac{A_{2} / A_{1}}{2^{n}(n-1) !}\right.\right.\right. \\
& \left.\left.\left.+\frac{A_{4} / A_{1}^{2}}{2^{n-2}(n-2) ! 4 !}\right)\right] w^{2 n}+O\left(\varepsilon^{3 / 2}\right)\right\} \\
= & \log \left\{e ^ { w ^ { 2 } / 2 } \left[1+e^{-w^{2} / 2} \varepsilon\left\{\sum_{n=1}^{\infty} \frac{A_{2} / A_{1}}{2^{n}(n-1) !} w^{2 n}\right.\right.\right. \\
& \left.\left.\left.+\sum_{n=2}^{\infty} \frac{A_{4} / A_{1}^{2}}{2^{n-2}(n-2) ! 4 !} w^{2 n}\right\}+O\left(\varepsilon^{3 / 2}\right)\right]\right\} \\
= & \frac{w^{2}}{2}+e^{-w^{2} / 2} \varepsilon\left[\sum_{n=0}^{\infty} \frac{A_{2} / A_{1}}{2^{n+1} n !} w^{2 n+2}+\sum_{n=0}^{\infty} \frac{A_{4} / A_{1}^{2}}{2^{n} n ! 4 !} w^{2 n+4}\right]+O\left(\varepsilon^{3 / 2}\right)
\end{aligned}
$$




$$
\begin{aligned}
& =\frac{w^{2}}{2}+\varepsilon\left(\frac{A_{2}}{2 A_{1}} w^{2}+\frac{A_{4}}{A_{1}^{2}} \frac{w^{4}}{4 !}\right)+O\left(\varepsilon^{3 / 2}\right) \\
& =\left(1+\varepsilon \frac{A_{2}}{A_{1}}\right) \frac{w^{2}}{2}+\varepsilon \frac{A_{4}}{A_{1}^{2}} \frac{w^{4}}{4 !}+O\left(\varepsilon^{3 / 2}\right) .
\end{aligned}
$$

Thus, referring to (3.15), we obtain the semi-invariants $\beta_{k}$ :

$$
\beta_{1}=0, \quad \beta_{2}=1+\varepsilon \frac{A_{2}}{A_{1}}, \quad \beta_{3}=0, \quad \beta_{4}=\varepsilon \frac{A_{4}}{A_{1}^{2}}, \quad \beta_{k}=0 \quad \text { for } \quad k \geq 5,
$$

where terms of order $\varepsilon^{3 / 2}$ have been neglected. Next we write

$$
\begin{aligned}
\psi_{\xi}(t) & =\exp \left[\sum_{k=1}^{\infty} \frac{\beta_{k}}{k !}(i t)^{k}\right] \\
& =\exp \left\{-\frac{t^{2}}{2}+\varepsilon\left[-\frac{A_{2}}{A_{1}} \frac{t^{2}}{2}+\frac{A_{4}}{A_{1}^{2}} \frac{t^{4}}{4 !}\right]+O\left(\varepsilon^{3 / 2}\right)\right\} \\
& =e^{-t^{2} / 2}\left\{1+\varepsilon\left[-\frac{A_{2}}{A_{1}} \frac{t^{2}}{2}+\frac{A_{4}}{A_{1}^{2}} \frac{t^{4}}{4 !}\right]\right\}+O\left(\varepsilon^{3 / 2}\right) .
\end{aligned}
$$

Then by comparing (4.13) with (3.13) we determine the coefficients $c_{k}$ :

$$
c_{2}=\frac{A_{2}}{A_{1}} \varepsilon, \quad c_{3}=0, \quad c_{4}=\frac{A_{4}}{A_{1}^{2}} \varepsilon, \quad c_{k}=0 \quad \text { for } \quad k \geq 5,
$$

where again terms of order $\varepsilon^{3 / 2}$ have been neglected. Finally, from (3.11) we have

$$
\begin{aligned}
F_{\xi}(u) & =\Phi(u)-\frac{\varepsilon}{\sqrt{2 \pi}} e^{-u^{2} / 2}\left\{\frac{A_{2} / A_{1}}{2 !} H_{1}(u)+\frac{A_{4} / A_{1}^{2}}{4 !} H_{3}(u)\right\}+O\left(\varepsilon^{3 / 2}\right) \\
& =\frac{1}{\sqrt{2 \pi}} \int_{-\infty}^{u} e^{-t^{2} / 2} d t-\frac{\varepsilon}{\sqrt{2 \pi}} e^{-u^{2} / 2}\left\{\frac{A_{2} / A_{1}}{2 !} u+\frac{A_{4} / A_{1}^{2}}{4 !}\left(u^{3}-3 u\right)\right\}+O\left(\varepsilon^{3 / 2}\right) .
\end{aligned}
$$

By differentiation we obtain the density function

$$
p_{\xi}(u)=\phi(u)\left\{1+\varepsilon\left[\frac{A_{2} / A_{1}}{2 !}\left(u^{2}-1\right)+\frac{A_{4} / A_{1}^{2}}{4 !}\left(u^{4}-6 u^{2}+3\right)\right]+O\left(\varepsilon^{3 / 2}\right)\right\},
$$

where $\phi(u)$ is given by $(3.23)$.

Let us now consider the calculation of the coefficient $A_{2}$ in (4.1). This involves a determination of the contributions of order $\varepsilon^{2}$ in (2.34). First consider the integral $J_{1}$, which is given by

$$
J_{1}=\iint_{T_{1}} G\left(x, z-\frac{s}{2}\right) G\left(x, z+\frac{s}{2}\right)\left\langle g_{1}\left(z-\frac{s}{2}\right) g_{1}\left(z+\frac{s}{2}\right)\right\rangle d s d z,
$$

where the triangle $T_{1}$ is indicated in Fig. 1 ; note that the area of $T_{1}$ is $\varepsilon^{2} / 4$. Thus, if we expand the integrand in powers of $s$ and $z$ about the point $z=1, s=0$, we need keep only the first, or constant, term in the expansion in order to obtain the $\varepsilon^{2}$ contribution to $J_{1}$. 
The result is

$$
J_{1}=\frac{\varepsilon^{2}}{4} G^{2}(x, 1)\left\langle g_{1}^{2}(1)\right\rangle+O\left(\varepsilon^{3}\right)
$$

where $\left\langle g_{1}^{2}(1)\right\rangle$ is found by evaluating the expression

$$
\left\langle g_{1}^{2}(z)\right\rangle=\sigma_{f}^{2}+\sigma_{q}^{2} y_{0}^{2}(z)+\sigma_{p}^{2} y_{0}^{\prime \prime 2}(z)-\sigma_{p}^{2} \rho_{p}^{\prime \prime}(0) y_{0}^{\prime 2}(z)
$$

for $z=1$. Eq. (4.19) follows from (2.31a) by introducing the variables $s$ and $z$, and then setting $s=0$. The integral $J_{2}$ over the triangle $T_{2}$ is calculated in a similar way:

$$
J_{2}=\frac{\varepsilon^{2}}{4} G^{2}(x, 0)\left\langle g_{1}^{2}(0)\right\rangle+O\left(\varepsilon^{3}\right)
$$

where $\left\langle g_{1}^{2}(0)\right\rangle$ is also obtained from (4.19).

There may also be a contribution of order $\varepsilon^{2}$ arising from the main integral in (2.34). This contribution can be found by expanding the integrand in (2.34) in powers of $s$ and keeping the linear, as well as the constant, term. The calculation is rendered somewhat more complicated by the fact that the derivative of one of the Green's function factors is discontinuous when $z \pm(s / 2)=x$. Thus we must consider the interval $|z-x| \leq \varepsilon / 2$ separately, so that

$$
\begin{aligned}
\int_{0}^{1} \int_{0}^{\varepsilon} G\left(x, z-\frac{s}{2}\right) G\left(x, z+\frac{s}{2}\right)\left\langle g_{1}\left(z-\frac{s}{2}\right)\right. & \left.g_{1}\left(z+\frac{s}{2}\right)\right\rangle d s d z \\
& =\int_{0}^{1} \int_{|z-x|>\varepsilon / 2}^{\varepsilon} \cdots d s d z+\int_{x-\varepsilon / 2}^{x+\varepsilon / 2} \int_{0}^{\varepsilon} \cdots d s d z .
\end{aligned}
$$

For $|z-x|>\varepsilon / 2$, we can write

$$
\begin{aligned}
& G\left(x, z+\frac{s}{2}\right)=G(x, z)+G^{\prime}(x, z) \frac{s}{2}+O\left(s^{2}\right), \\
& G\left(x, z-\frac{s}{2}\right)=G(x, z)-G^{\prime}(x, z) \frac{s}{2}+O\left(s^{2}\right)
\end{aligned}
$$

where $G^{\prime}$ refers to the derivative with respect to the second argument of $G$. Then

$$
G\left(x, z+\frac{s}{2}\right) G\left(x, z-\frac{s}{2}\right)=G^{2}(x, z)+O\left(s^{2}\right)
$$

By starting from (2.31a) and proceeding in a similar way it is possible to show that

$$
\left\langle g_{1}\left(z+\frac{s}{2}\right) g_{1}\left(z-\frac{s}{2}\right)\right\rangle=\sigma_{f}^{2}+\sigma_{q}^{2} y_{0}^{2}(z)+\sigma_{p}^{2} y_{0}^{\prime \prime 2}(z)-\sigma_{p}^{2} \rho_{p}^{\prime \prime}(0) y_{0}^{\prime 2}(z)+O\left(s^{2}\right) \text {. }
$$

For example, consider the second term on the right side of (2.31a):

$$
\begin{aligned}
\sigma_{q}^{2} \rho_{q}\left(x_{2}-x_{1}\right) y_{0}\left(x_{1}\right) y_{0}\left(x_{2}\right) & =\sigma_{q}^{2} \rho_{q}(s) y_{0}\left(z-\frac{s}{2}\right) y_{0}\left(z+\frac{s}{2}\right) \\
= & \sigma_{q}^{2}\left[1+O\left(s^{2}\right)\right]\left[y_{0}(z)-\frac{s}{2} y_{0}^{\prime}(z)+O\left(s^{2}\right)\right]\left[y_{0}(z)+\frac{s}{2} y_{0}^{\prime}(z)+O\left(s^{2}\right)\right] \\
= & \sigma_{q}^{2} y_{0}^{2}(z)+O\left(s^{2}\right) .
\end{aligned}
$$


The other terms on the right side of (4.24) are obtained in a like manner. Thus

$$
\begin{aligned}
\int_{0|z-x|>\varepsilon / 2}^{1} \int_{0}^{\varepsilon} G(x, & \left.z-\frac{s}{2}\right) G\left(x, z+\frac{s}{2}\right)\left\langle g_{1}\left(z-\frac{s}{2}\right) g_{1}\left(z+\frac{s}{2}\right)\right\rangle d s d z \\
& =\int_{0}^{1} \int_{|z-x|>\varepsilon / 2}^{\varepsilon}\left[G^{2}(x, z)\left\langle g_{1}^{2}(z)\right\rangle+O\left(s^{2}\right)\right] d s d z \\
& =\varepsilon\left(\int_{0}^{x-\varepsilon / 2}+\int_{x+\varepsilon / 2}^{1}\right) G^{2}(x, z)\left\langle g_{1}^{2}(z)\right\rangle d z+O\left(\varepsilon^{3}\right) \\
& =\varepsilon \int_{0}^{1} G^{2}(x, z)\left\langle g_{1}^{2}(z)\right\rangle d z-\varepsilon \int_{x-\varepsilon / 2}^{x+\varepsilon / 2} G^{2}(x, z)\left\langle g_{1}^{2}(z)\right\rangle d z+O\left(\varepsilon^{3}\right) .
\end{aligned}
$$

Upon substituting (4.26) into (4.21) we are led to consider the combination

$$
\begin{array}{r}
\int_{x-\varepsilon / 2}^{x+\varepsilon / 2} \int_{0}^{\varepsilon} G\left(x, z-\frac{s}{2}\right) G\left(x, z+\frac{s}{2}\right)\left\langle g_{1}\left(z-+\frac{s}{2}\right) g_{1}\left(z+\frac{s}{2}\right)\right\rangle d s d z \\
-\varepsilon \int_{x-\varepsilon / 2}^{x+\varepsilon / 2} G^{2}(x, z)\left\langle g_{1}^{2}(z)\right\rangle d z
\end{array}
$$

By the mean-value theorem, this combination can be expressed as

$$
\varepsilon \int_{x-\varepsilon / 2}^{x+\varepsilon / 2}\left[G\left(x, z-\frac{\hat{s}}{2}\right) G\left(x, z+\frac{\hat{s}}{2}\right)\left\langle g_{1}\left(z-\frac{\hat{s}}{2}\right) g_{1}\left(z+\frac{\hat{s}}{2}\right)\right\rangle-G^{2}(x, z)\left\langle g_{1}^{2}(z)\right\rangle\right] d z
$$

where $0<\hat{s}<\varepsilon$. Even though $G^{\prime}(x, t)$ is not continuous when $t=x$, the one-sided derivatives of $G$ are bounded throughout the region under consideration, and therefore

$$
\left|G\left(x, z \pm \frac{\hat{s}}{2}\right)-G(x, z)\right| \leq \bar{G} \varepsilon
$$

where

$$
\bar{G}=\sup \left|G^{\prime}(x, z)\right|, \quad 0 \leq x, z \leq 1 ;
$$

at a discontinuity point, $G^{\prime}$ refers to a one-sided derivative. Further, from (4.19) and (4.24) it follows that

$$
\left|\left\langle g_{1}\left(z-\frac{\hat{s}}{2}\right) g_{1}\left(z+\frac{\hat{s}}{2}\right)\right\rangle-\left\langle g_{1}^{2}(z)\right\rangle\right|=O\left(\varepsilon^{2}\right) .
$$

By adding and subtracting suitable quantities to the integrand in (4.28), using the triangle inequality, and referring to (4.29) and (4.31), we find that the integrand in (4.28) is of order $\varepsilon$. Consequently, the expression in (4.28) is of order $\varepsilon^{3}$. Thus, from (4.21) and (4.26),

$$
\begin{aligned}
\int_{0}^{1} \int_{0}^{\varepsilon} G\left(x, z-\frac{s}{2}\right) G\left(x, z+\frac{s}{2}\right)\left\langle g_{1}\left(z-\frac{s}{2}\right)\right. & \left.g_{1}\left(z+\frac{s}{2}\right)\right\rangle d s d z \\
& =\varepsilon \int_{0}^{1} G^{2}(x, z)\left\langle g_{1}^{2}(z)\right\rangle d z+O\left(\varepsilon^{3}\right)
\end{aligned}
$$


that is, this integral has no terms of order $\varepsilon^{2}$. Finally, going back to (2.34), we have

$$
\begin{aligned}
\left\langle y_{1}^{2}(x)\right\rangle=I_{2}(x)=2 \varepsilon \int_{0}^{1} G^{2}(x, z) & \left\langle g_{1}^{2}(z)\right\rangle d z \\
& \quad-\frac{\varepsilon^{2}}{2}\left[G^{2}(x, 1)\left\langle g_{1}^{2}(1)\right\rangle+G^{2}(x, 0)\left\langle g_{1}^{2}(0)\right\rangle\right]+O\left(\varepsilon^{3}\right),
\end{aligned}
$$

where $\left\langle g_{1}^{2}(z)\right\rangle$ is given by (4.18). Thus $A_{1}(x)$ and $A_{2}(x)$ in (4.1) are the coefficients of $\varepsilon$ and $\varepsilon^{2}$ respectively in (4.33); of course, $A_{1}(x)$ is the same as given by (2.35). The preceding derivation assumes tacitly that $x$ is not within a distance $\varepsilon / 2$ of either endpoint. If this is not so, then the argument must be modified slightly, but the result is the same.

Now we turn to the calculation of the coefficient $A_{4}$ in (4.3). From (2.37) and (2.38) we have

$$
\begin{aligned}
I_{4}(x)=\iiint \int_{R_{4}} G\left(x, x_{1}\right) \cdots G(x, & \left.x_{4}\right)[ \\
{[} & \left\langle g_{1}\left(x_{1}\right) \cdots g_{1}\left(x_{4}\right)\right\rangle \\
-3 & \left.\left\langle g_{1}\left(x_{1}\right) g_{1}\left(x_{2}\right)\right\rangle\left\langle g_{1}\left(x_{3}\right) g_{1}\left(x_{4}\right)\right\rangle\right] d x_{1} \cdots d x_{4},
\end{aligned}
$$

where the region of integration is that portion of the four-dimensional cube $0 \leq x_{1}, x_{2}$, $x_{3}, x_{4} \leq 1$ satisfying

$$
\left|x_{1}-x_{2}\right| \leq \varepsilon, \quad\left|x_{2}-x_{3}\right| \leq \varepsilon, \quad \text { and } \quad\left|x_{3}-x_{4}\right| \leq \varepsilon,
$$

or one of eleven other sets of inequalities obtained from (4.35) by permuting the variables. If we remove the absolute value bars, then we can replace (4.35) by twenty-four sets of inequalities, of which

$$
0 \leq x_{1}-x_{2} \leq \varepsilon, \quad 0 \leq x_{2}-x_{3} \leq \varepsilon, \quad 0 \leq x_{3}-x_{4} \leq \varepsilon
$$

is typical. The integration region is a portion of a cylinder whose axis is the line $x_{1}=$ $x_{2}=x_{3}=x_{4}$. It is convenient to introduce new coordinates $s_{1}, s_{2}, s_{3}, s_{4}$ by the transformation

$$
\begin{array}{ll}
s_{1}=\left(x_{1}+x_{2}+x_{3}+x_{4}\right) / 2, & s_{2}=\left(x_{1}-x_{2}+x_{3}-x_{4}\right) / 2, \\
s_{3}=\left(x_{1}+x_{2}-x_{3}-x_{4}\right) / 2, & s_{4}=\left(x_{1}-x_{2}-x_{3}+x_{4}\right) / 2,
\end{array}
$$

or its inverse

$$
\begin{array}{ll}
x_{1}=\left(s_{1}+s_{2}+s_{3}+s_{4}\right) / 2, & x_{2}=\left(s_{1}-s_{2}+s_{3}-s_{4}\right) / 2, \\
x_{3}=\left(s_{1}+s_{2}-s_{3}-s_{4}\right) / 2, & x_{4}=\left(s_{1}-s_{2}-s_{3}+s_{4}\right) / 2 .
\end{array}
$$

The $s_{1} s_{2} s_{3} s_{4}$-coordinate system is an orthogonal system, and is oriented so that the $s_{1}$-axis lies along the axis of the cylinder. A straightforward calculation shows that

$$
\frac{\partial\left(s_{1}, s_{2}, s_{3}, s_{4}\right)}{\partial\left(x_{1}, x_{2}, x_{3}, x_{4}\right)}=\frac{\partial\left(x_{1}, x_{2}, x_{3}, x_{4}\right)}{\partial\left(s_{1}, s_{2}, s_{3}, s_{4}\right)}=1 .
$$

To find the part of $I_{4}$ that is of lowest order in $\varepsilon$ we can expand the integrand in the transverse variables and then keep only the first term. This amounts to evaluating the integrand on the $s_{1}$-axis, so that

$$
\begin{aligned}
I_{4}(x)=V \int_{0}^{2} G^{4}\left(x, \frac{s_{1}}{2}\right)\left\langle g_{1}^{4}\left(\frac{s_{1}}{2}\right)\right\rangle d s_{1} & \\
& -3 \bar{V} \int_{0}^{2} G^{4}\left(x, \frac{s_{1}}{2}\right)\left\langle g_{1}^{2}\left(\frac{s_{1}}{2}\right)\right\rangle^{2} d s_{1}+O\left(\varepsilon^{4}\right),
\end{aligned}
$$


where $V$ is the volume of the three-dimensional cross-section of the cylindrical integration region $R_{4}$, and $\bar{V}$ is the volume of the cross-section of the smaller cylinder $\left(R_{2} \times R_{2}\right) \cap$ $R_{4}$. The cross-section of $R_{4}$ is a star-shaped region with twenty-four points, as shown in Fig. 3. It is bounded by twelve identical plane faces, one of which is shaded in the figure. The inequality sets typified by (4.36) divide the region into twenty-four congruent subregions, each of which is a parallelepiped. For example, in an $s_{2} s_{3} s_{4}$-subspace corresponding to $s_{1}=$ constant, the inequalities (4.36) yield the parallelepiped with vertices

$$
\begin{gathered}
(0,0,0), \quad(0, \varepsilon, 0), \quad(\varepsilon, \varepsilon, 0), \quad(\varepsilon, 2 \varepsilon, 0), \quad(\varepsilon / 2, \varepsilon / 2, \varepsilon / 2), \\
(\varepsilon / 2, \varepsilon / 2,-\varepsilon / 2), \quad(\varepsilon / 2,3 \varepsilon / 2, \varepsilon / 2), \quad(\varepsilon / 2,3 \varepsilon / 2,-\varepsilon / 2) .
\end{gathered}
$$

The volume of this parallelepiped is readily found to be $\varepsilon^{3} / 2$, so the volume of the entire cross-section is $12 \varepsilon^{3}$. A further examination reveals that $\bar{V}=V / 2$. Substituting these values in (4.39) and letting $z=s_{1} / 2$ to normalize the interval of integration, we finally obtain

$$
I_{4}(x)=24 \varepsilon^{3} \int_{0}^{1} G^{4}(x, z)\left[\left\langle g_{1}^{4}(z)\right\rangle-\frac{3}{2}\left\langle g_{1}^{2}(z)\right\rangle^{2}\right] d z+O\left(\varepsilon^{4}\right),
$$

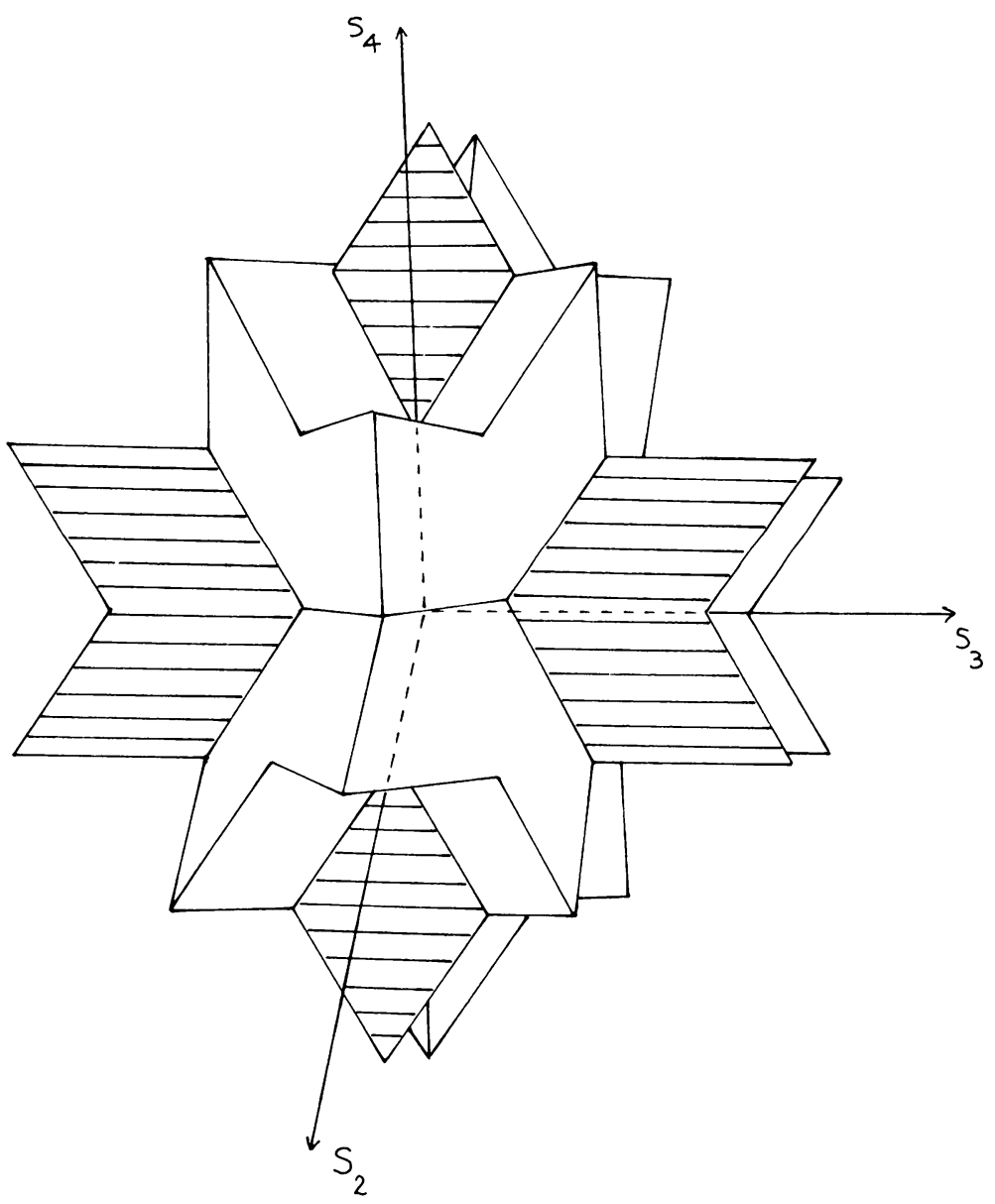

Fig. 3. 
so

$$
A_{4}(x)=24 \int_{0}^{1} G^{4}(x, z)\left[\left\langle g_{1}^{4}(z)\right\rangle-\frac{3}{2}\left\langle g_{1}^{2}(z)\right\rangle^{2}\right] d z
$$

5. Example. To illustrate the application of the results derived in the preceding sections, consider the example

$$
-y^{\prime \prime}+\eta q_{1}(x, \omega) y=x+\eta f_{1}(x, \omega), \quad y(0)=y(1)=0 .
$$

In the notation of Sec. 2 we have

$$
p_{0}(x)=1, \quad p_{1}(x, \omega)=0, \quad q_{0}(x)=0, \quad f_{0}(x)=x ;
$$

the statistical properties of $q_{1}$ and $f_{1}$ will be specified later. The coefficient $p(x, \omega)$ was chosen to be nonrandom in order to simplify the necessary calculations. As in Sec. 2 we assume that

$$
y(x, \omega)=y_{0}(x)+\eta y_{1}(x, \omega)+O\left(\eta^{2}\right),
$$

whereupon it follows that $y_{0}$ satisfies

$$
L_{0}\left[y_{0}\right] \equiv-y_{0}^{\prime \prime}=x, \quad y(0)=y(1)=0 .
$$

The Green's function for $L_{0}$ with the given boundary conditions is

$$
\begin{aligned}
G\left(x, x_{1}\right) & =x_{1}(1-x), & & 0 \leq x_{1} \leq x \\
& =x\left(1-x_{1}\right), & & x \leq x_{1} \leq 1
\end{aligned}
$$

and

$$
y_{0}(x)=\int_{0}^{1} G\left(x, x_{1}\right) f_{0}\left(x_{1}\right) d x_{1}=\frac{1}{6}\left(x-x^{3}\right)
$$

Further,

$$
y_{1}(x, \omega)=\int_{0}^{1} G\left(x, x_{1}\right) g_{1}\left(x_{1}, \omega\right) d x_{1},
$$

where

$$
g_{1}(x, \omega)=f_{1}(x, \omega)-q_{1}(x, \omega) y_{0}(x) .
$$

To estimate the distribution function of $y_{1}(x, \omega)$ the first step is to normalize $y_{1}$ by calculating $A_{1}(x)$ from $(2.35 \mathrm{~b})$. Since $p_{1}(x, \omega) \equiv 0$, we have

$$
\begin{aligned}
A_{1}(x) & =2 \int_{0}^{1} G^{2}(x, z)\left[\sigma_{f}^{2}+\sigma_{q}^{2} y_{0}^{2}(z)\right] d z \\
& =\frac{2}{3} \sigma_{f}^{2} x^{2}(1-x)^{2}+\frac{1}{18} \sigma_{q}^{2} x^{2}\left(\frac{23}{1260}-\frac{2 x^{3}}{15}+\frac{x^{4}}{10}+\frac{4 x^{5}}{35}-\frac{2 x^{6}}{21}-\frac{2 x^{7}}{63}+\frac{x^{8}}{36}\right) .
\end{aligned}
$$

Next we use (3.29) and (3.30) to determine $A_{3}(x)$; this requires information, or assumptions, about $\left\langle q_{1}^{3}\right\rangle$ and $\left\langle f_{1}^{3}\right\rangle$. If it turns out that $A_{3}(x) \neq 0$, then (3.22) gives the density 
function $p_{\xi}$ up to terms of order $\sqrt{ } \varepsilon$. Let us suppose, however, that $\left\langle q_{1}^{3}\right\rangle=\left\langle f_{1}^{3}\right\rangle=0$ so that $A_{3}(x)=0$. In this case we wish to use (4.16) to estimate $p_{\xi}$, and this requires the evaluation of $A_{2}(x)$ and $A_{4}(x)$ from (4.33) and (4.41) respectively. Since $G(x, 0)=G(x, 1)=0$ from (5.5), it follows at once from (4.33) that $A_{2}(x)=0$.

The analytical calculation of $A_{4}(x)$ from (4.41), while not difficult in principle, requires a rather lengthy integration process, which ultimately yields a polynomial of degree twenty whose coefficients depend on $\left\langle f_{1}^{4}\right\rangle, \sigma_{f}^{2} \sigma_{q}^{2}$, and $\left\langle q_{1}^{4}\right\rangle$. In most cases it is probably better to evaluate $A_{4}(x)$ numerically for those values of $x$ that are of interest.

Since $A_{2}(x)=0$ in this example, the expression (4.16) for $p_{5}(u)$ reduces to

$$
\begin{aligned}
p_{\zeta}(u) & =\phi(u)+\varepsilon C e^{-u^{2} / 2}\left(u^{4}-6 u^{2}+3\right)+O\left(\varepsilon^{3 / 2}\right) \\
& =\phi(u)+\varepsilon \phi_{1}(u)+O\left(\varepsilon^{3 / 2}\right),
\end{aligned}
$$

where $C(x)=A_{4}(x) / 24 \sqrt{2 \pi} A_{1}^{2}(x)$. In the accompanying plots we have chosen $x=0.5$ and have assumed that

$$
\sigma_{f}^{2}=\sigma_{q}^{2}=1.0 ; \quad\left\langle f_{1}^{4}\right\rangle=1.75 ; \quad\left\langle q_{1}^{4}\right\rangle=2.0 .
$$

The solid and dashed curves in Fig. 4 show the normal density function $\phi(u)$ and the correction term $\phi_{1}(u)$ respectively. The curves in Fig. 5 are plots of $p_{\xi}(u)$ from $(5.10)$ when $\varepsilon=0,0.1$, and 0.5 respectively. In this example, at least, the actual density function is close to the normal even for fairly large values of $\varepsilon$.

6. The corresponding eigenvalue problem. Purkert and vom Scheidt $[5,7]$ have established properties similar to those in Sec. 2 for a large class of eigenvalue problems.

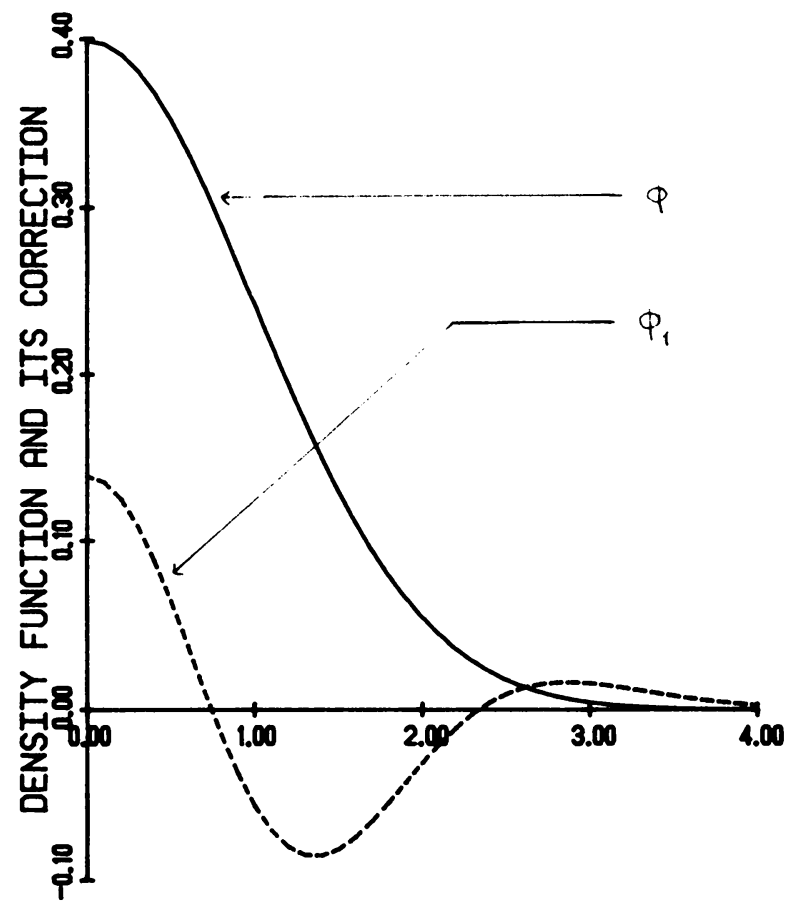

F1G. 4. 


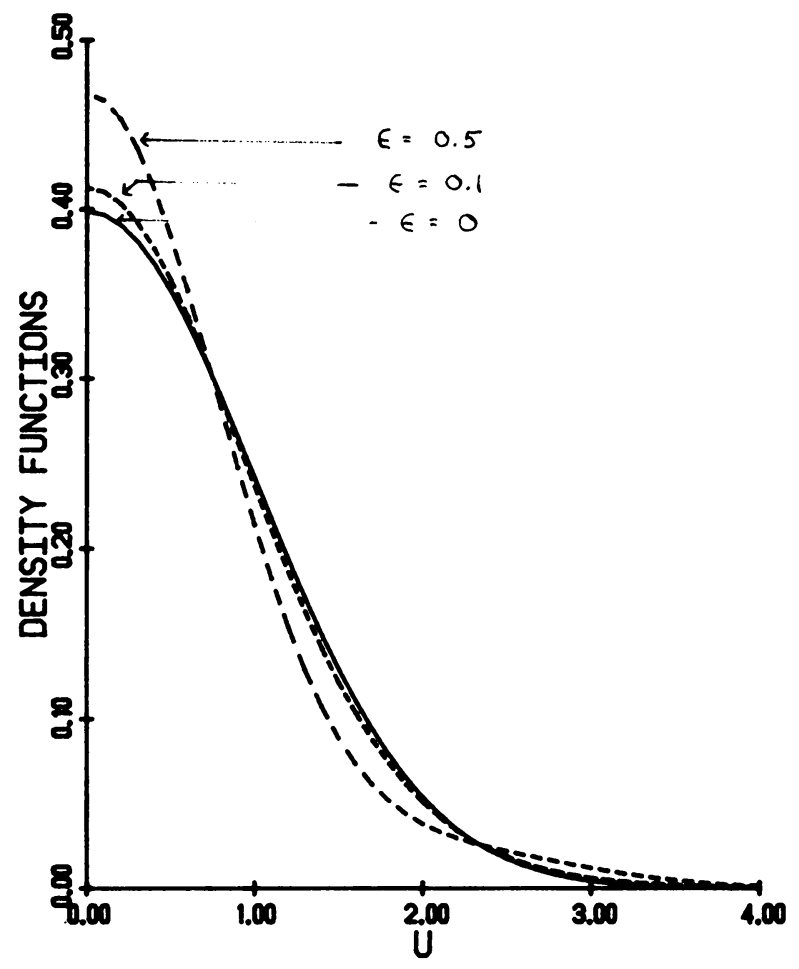

Fig. 5 .

Here we describe briefly the extension of the results in Secs. 3 and 4 to this kind of problem. For simplicity we consider an eigenvalue problem analogous to the boundaryvalue problem of Sec. 2 ; it will be apparent that the derivation can be extended to a much larger class of problems, such as that investigated in [5]. Suppose that

$$
\begin{gathered}
L[y]=-\left[p(x, \omega) y^{\prime}\right]^{\prime}+q(x, \omega) y=\lambda r(x, \omega) y, \quad 0<x<1 \\
U_{i}[y]=0 ; \quad i=1,2 .
\end{gathered}
$$

We assume that the coefficients $p(x, \omega)$ and $q(x, \omega)$ satisfy the conditions given in Sec. 2 , and that the boundary conditions again have the form (2.3). The coefficient $r(x, \omega)$ is continuous and positive on $0 \leq x \leq 1$ except possibly for an $\omega$-set of probability zero. We assume that $p(x, \omega)$ and $q(x, \omega)$ again have the form $(2.4 \mathrm{a}, \mathrm{b})$, and that

$$
r(x, \omega)=r_{0}(x)+\eta r_{1}(x, \omega), \quad r_{0}(x)=\langle r(x, \omega)\rangle,
$$

where $r_{0}(x)>0$ on $0 \leq x \leq 1$. The random perturbations $p_{1}, q_{1}$, and $r_{1}$ have mean zero, and we assume them to be pairwise independent and wide-sense stationary with correlation length $\varepsilon$. Thus $p_{1}$ and $q_{1}$ satisfy (2.7) and (2.8) respectively, while

$$
\begin{aligned}
\left\langle r_{1}\left(x_{1}\right) r_{1}\left(x_{2}\right)\right\rangle & =0, & & \left|x_{2}-x_{1}\right|>\varepsilon \\
& =\sigma_{r}^{2} \rho_{r}\left(x_{2}-x_{1}\right), & & \left|x_{2}-x_{1}\right| \leq \varepsilon .
\end{aligned}
$$

Under the given assumptions the problem (6.1), (6.2) has a sequence of eigenvalues 
$\left\{\lambda_{i}(\omega)\right\}$ and corresponding eigenfunctions $\left\{y_{i}(x, \omega)\right\}$. To determine them we assume that the $n$th eigenvalue and eigenfunction have the form

$$
\lambda_{n}=\sum_{j=0}^{\infty} \lambda_{n j} \eta^{j}, \quad y_{n}(x)=\sum_{j=0}^{\infty} y_{n j}(x) \eta^{j}
$$

A straightforward perturbation analysis leads to the problems

$$
-\left(p_{0} y_{n 0}^{\prime}\right)^{\prime}+q_{0} y_{n 0}=\lambda_{n 0} r_{0} y_{n 0}, \quad U_{i}\left[y_{n 0}\right]=0
$$

and

$$
\begin{aligned}
-\left(p_{0} y_{n 1}^{\prime}\right)^{\prime}+q_{0} y_{n 1}-\lambda_{n 0} r_{0} y_{n 1} & =\left(p_{1} y_{n 0}^{\prime}\right)^{\prime} \\
& -q_{1} y_{n 0}+\lambda_{n 0} r_{1} y_{n 0}+\lambda_{n 1} r_{0} y_{n 0}, \quad U_{i}\left[y_{n 1}\right]=0
\end{aligned}
$$

for $y_{n 0}, \lambda_{n 0}$ and $y_{n 1}, \lambda_{n 1}$ respectively. Let us denote the $n$th eigenvalue of (6.6) by $\mu_{n}$ and the corresponding eigenfunction, normalized with respect to the weight function $r_{0}$, by $w_{n}$. Then (6.7) has a solution if and only if the right side of the differential equation is orthogonal to $w_{n}$. From this condition we find that

$$
\lambda_{n 1}(\omega)=\int_{0}^{1} h_{n}(x, \omega) w_{n}(x) d x,
$$

where

$$
h_{n}(x, \omega)=-\left[p_{1}(x, \omega) w_{n}^{\prime}(x)\right]^{\prime}+q_{1}(x, \omega) w_{n}(x)-\mu_{n} r_{1}(x, \omega) w_{n}(x) .
$$

If (6.8) is satisfied, then $y_{n 1}$ can be found from (6.7) by means of an eigenfunction expansion

$$
y_{n 1}(x, \omega)=\sum_{\substack{i=1 \\ i \neq n}}^{\infty} \frac{a_{n i}(\omega)}{\mu_{i}-\mu_{n}} w_{i}(x),
$$

where

$$
a_{n i}(\omega)=\int_{0}^{1} h_{n}(x, \omega) w_{i}(x) d x, \quad i \neq n .
$$

An analysis similar to that in Secs. 2, 3, and 4 can be based on (6.8) through (6.11).

If we compare (6.8) with (2.18) we see that they are of the same form with $h_{n}(x, \omega)$ corresponding to $g_{1}\left(x_{1}, \omega\right)$ and $w_{n}(x)$ to $G_{1}\left(x, x_{1}\right)$. Thus we can obtain estimates for the distribution function of $\lambda_{n 1}$ similar to (3.21) and (4.15), provided that we can determine the coefficients corresponding to $A_{1}, A_{2}, A_{3}$, and $A_{4}$. To do this, define $I_{2}^{*}, I_{3}^{*}$, and $I_{4}^{*}$ analogously to $I_{2}, I_{3}$, and $I_{4}$, respectively. To evaluate $I_{2}^{*}$ we again introduce the variables $z$ and $s$ by (2.32) so that

$$
I_{2}^{*}=2 \int_{0}^{1} \int_{0}^{e}\left\langle h_{n}\left(z-\frac{s}{2}\right) h_{n}\left(z+\frac{s}{2}\right)\right\rangle w_{n}\left(z-\frac{s}{2}\right) w_{n}\left(z+\frac{s}{2}\right) d s d z-2\left(J_{1}^{*}+J_{2}^{*}\right),
$$

where $J_{1}^{*}$ and $J_{2}^{*}$ are the integrals of the same integrand over the triangles $T_{1}$ and $T_{2}$ in Fig. 1. To evaluate the main integral up to terms of order $\varepsilon^{2}$ we expand the integrand 
in powers of $s$ and keep the first two terms. This requires that $h_{n}$ be differentiable, and this in turn requires the additional hypothesis that $p_{1}^{\prime}, q_{1}$, and $r_{1}$ be differentiable with probability one. Assuming this to be true, we have

$$
\begin{aligned}
h_{n}\left(z-\frac{s}{2}\right) h_{n}\left(z+\frac{s}{2}\right) & =\left[h_{n}(z)-\frac{s}{2} h_{n}^{\prime}(z)+O\left(s^{2}\right)\right]\left[h_{n}(z)+\frac{s}{2} h_{n}^{\prime}(z)+O\left(s^{2}\right)\right] \\
& =h_{n}^{2}(z)+O\left(s^{2}\right)
\end{aligned}
$$

and similarly for $w_{n}(z-s / 2) w_{n}(z+s / 2)$. Since there is no term of order $s$ in (6.13), it follows that there is no $\varepsilon^{2}$ term in the main integral. To evaluate $J_{1}^{*}$ and $J_{2}^{*}$ we need keep only the first term in the expansion of the integrand in $s$, since the area of the triangular region is $\varepsilon^{2} / 4$ in each case. The result is

$$
\begin{aligned}
I_{2}^{*} & =2 \varepsilon \int_{0}^{1}\left\langle h_{n}^{2}(z)\right\rangle w_{n}^{2}(z) d z-\frac{\varepsilon^{2}}{2}\left[\left\langle h_{n}^{2}(0)\right\rangle w_{n}^{2}(0)+\left\langle h_{n}^{2}(1)\right\rangle w_{n}^{2}(1)\right]+O\left(\varepsilon^{3}\right), \\
& =A_{1}^{*} \varepsilon+A_{2}^{*} \varepsilon^{2}+O\left(\varepsilon^{3}\right) .
\end{aligned}
$$

To obtain the integrand in (6.14) more explicitly, we can start from (6.9) and use some of the results of Sec. 2, thereby obtaining

$$
\left\langle h_{n}^{2}(z)\right\rangle=\sigma_{p}^{2} w_{n}^{\prime \prime 2}(z)-\sigma_{p}^{2} \rho_{p}^{\prime \prime}(0) w_{n}^{\prime 2}(z)+\sigma_{q}^{2} w_{n}^{2}(z)+\mu_{n}^{2} \sigma_{r}^{2} w_{n}^{2}(z) .
$$

In the same way as in Secs. 3 and 4 it follows that

and

$$
I_{3}^{*}=6 \varepsilon^{2} \int_{0}^{1}\left\langle h_{n}^{3}(z)\right\rangle w_{n}^{3}(z) d z+O\left(\varepsilon^{3}\right)=A_{3}^{*} \varepsilon^{2}+O\left(\varepsilon^{3}\right),
$$

$$
\begin{aligned}
I_{4}^{*} & =24 \varepsilon^{3} \int_{0}^{1}\left[\left\langle h_{n}^{4}(z)\right\rangle-\frac{3}{2}\left\langle h_{n}^{2}(z)\right\rangle^{2}\right] w_{n}^{4}(z) d z+O\left(\varepsilon^{4}\right) \\
& =A_{4}^{*} \varepsilon^{3}+O\left(\varepsilon^{4}\right) .
\end{aligned}
$$

Finally, we define the normalized random variable $\zeta$ by

$$
\zeta=\lambda_{1} / \sqrt{A_{1}^{*} \varepsilon} .
$$

Then (3.21) and (4.15) can be used to give the distribution function for $\zeta$ simply by replacing $A_{1}$ by $A_{1}^{*}, A_{2}$ by $A_{2}^{*}$, and so on.

The analysis of (6.10) and (6.11) for the eigenfunctions is similar, but is rendered more complicated in practice by the summation in (6.10). Let

$$
\hat{I}_{2}^{(i, j)}=\left\langle a_{n i} a_{n j}\right\rangle=\int_{0}^{1} \int_{0}^{1}\left\langle h_{n}\left(x_{1}\right) h_{n}\left(x_{2}\right)\right\rangle w_{i}\left(x_{1}\right) w_{j}\left(x_{2}\right) d x_{1} d x_{2} .
$$

Then

$$
\begin{aligned}
\hat{I}_{2}^{(i, j)}= & 2 \varepsilon \int_{0}^{1}\left\langle h_{n}^{2}(z)\right\rangle w_{i}(z) w_{j}(z) d z \\
& -\frac{\varepsilon^{2}}{2}\left[h_{n}^{2}(0) w_{i}(0) w_{j}(0)+h_{n}^{2}(1) w_{i}(1) w_{j}(1)\right]+O\left(\varepsilon^{3}\right) \\
= & \hat{A}_{1}^{(i, j)} \varepsilon+\hat{A}_{2}^{(i, j)} \varepsilon^{2}+O\left(\varepsilon^{3}\right),
\end{aligned}
$$


where $\left\langle h_{n}^{2}(z)\right\rangle$ is given by (6.15). From (6.10) we have

$$
\begin{aligned}
\hat{I}_{2}(x)=\left\langle y_{n 1}^{2}(x)\right\rangle & =\sum_{\substack{i=1 \\
i, j \neq n}}^{\infty} \sum_{\substack{j=1 \\
i}}^{\infty} \frac{\left\langle a_{n i} a_{n j}\right\rangle w_{i}(x) w_{j}(x)}{\left(\mu_{i}-\mu_{n}\right)\left(\mu_{j}-\mu_{n}\right)} \\
& =\hat{A}_{1}(x) \varepsilon+\hat{A}_{2}(x) \varepsilon^{2}+O\left(\varepsilon^{3}\right),
\end{aligned}
$$

where

$$
\hat{A}_{1}(x)=\sum_{i=1}^{\infty} \sum_{\substack{i=1 \\ i, j \neq n}}^{\infty} \frac{\hat{A}_{1}^{(i, j)} w_{i}(x) w_{j}(x)}{\left(\mu_{i}-\mu_{n}\right)\left(\mu_{j}-\mu_{n}\right)}
$$

and $\hat{A}_{2}(x)$ is given by a similar expression. In much the same way

$$
\hat{I}_{3}(x)=\left\langle y_{n 1}^{3}(x)\right\rangle=\hat{A}_{3}(x) \varepsilon^{2}+O\left(\varepsilon^{3}\right),
$$

where

$$
\hat{A}_{3}(x)=\sum_{\substack{i=1 \\ i, k, j \neq n}}^{\infty} \sum_{\substack{j=1 \\ k}}^{\infty} \sum_{k=1}^{\infty} \frac{\hat{A}_{3}^{(i, j, k)} w_{i}(x) w_{j}(x) w_{k}(x)}{\left(\mu_{i}-\mu_{n}\right)\left(\mu_{j}-\mu_{n}\right)\left(\mu_{k}-\mu_{n}\right)}
$$

and

$$
\hat{A}_{3}^{(i, j, k)}=6 \int_{0}^{1}\left\langle h_{n}^{3}(z)\right\rangle w_{i}(z) w_{j}(z) w_{k}(z) d z
$$

Also,

$$
\hat{I}_{4}(x)=\hat{A}_{4}(x) \varepsilon^{3}+O\left(\varepsilon^{4}\right)
$$

where

$$
\hat{A}_{4}(x)=\sum_{i=1}^{\infty} \sum_{\substack{j=1 \\ i, j, k, l \neq n}}^{\infty} \sum_{\substack{k=1 \\ l=1}}^{\infty} \frac{\hat{A}_{4}^{(i, j, k, l)} w_{i}(x) w_{j}(x) w_{k}(x) w_{l}(x)}{\left(\mu_{i}-\mu_{n}\right)\left(\mu_{j}-\mu_{n}\right)\left(\mu_{k}-\mu_{n}\right)\left(\mu_{l}-\mu_{n}\right)}
$$

and

$$
\hat{A}_{4}^{(i, j, k, l)}=24 \int_{0}^{1}\left[\left\langle h_{n}^{4}(z)\right\rangle-\frac{3}{2}\left\langle h_{n}^{2}(z)\right\rangle^{2}\right] w_{i}(z) w_{j}(z) w_{k}(z) w_{l}(z) d z .
$$

Finally we define the normalized random variable

$$
v_{n}(x, \omega)=y_{n 1}(x, \omega) / \sqrt{\hat{A}_{1}(x) \varepsilon} .
$$

Then (3.21) and (4.15) can be used again to estimate the distribution function for $v_{n}(x)$ provided that $A_{1}$ is replaced by $\hat{A}_{1}$ and so forth.

Note added in proof: We mention here two extensions of the results in this paper that were obtained after the preparation of the original manuscript.

First, suppose that the coefficients $p, q$, and $f$ are weakly correlated but not necessarily 
stationary. Then (2.7) through (2.9) are replaced by

$$
\begin{aligned}
\left\langle p_{1}\left(x_{1}\right) p_{1}\left(x_{2}\right)\right\rangle & =0, & & \left|x_{2}-x_{1}\right|>\varepsilon ; \\
& =R_{p}\left(x_{1}, x_{2}\right), & & \left|x_{2}-x_{1}\right| \leq \varepsilon ; \\
\left\langle q_{1}\left(x_{1}\right) q_{1}\left(x_{2}\right)\right\rangle & =0, & & \left|x_{2}-x_{1}\right|>\varepsilon ; \\
& =R_{q}\left(x_{1}, x_{2}\right), & & \left|x_{2}-x_{1}\right| \leq \varepsilon ; \\
\left\langle f_{1}\left(x_{1}\right) f_{1}\left(x_{2}\right)\right\rangle & =0, & & \left|x_{2}-x_{1}\right|>\varepsilon ; \\
& =R_{f}\left(x_{1}, x_{2}\right), & & \left|x_{2}-x_{1}\right| \leq \varepsilon .
\end{aligned}
$$

It follows that

$$
\begin{aligned}
\left\langle p_{1}\left(x_{1}\right) p_{1}^{\prime}\left(x_{2}\right)\right\rangle & =0, & & \left|x_{2}-x_{1}\right|>\varepsilon ; \\
& =\partial_{2} R_{p}\left(x_{1}, x_{2}\right), & & \left|x_{2}-x_{1}\right| \leq \varepsilon ; \\
\left\langle p_{1}^{\prime}\left(x_{1}\right) p_{1}\left(x_{2}\right)\right\rangle & =0, & & \left|x_{2}-x_{1}\right|>\varepsilon ; \\
& =\partial_{1} R_{p}\left(x_{1}, x_{2}\right), & & \left|x_{2}-x_{1}\right| \leq \varepsilon ; \\
\left\langle p_{1}^{\prime}\left(x_{1}\right) p_{1}^{\prime}\left(x_{2}\right)\right\rangle & =0, & & \left|x_{2}-x_{1}\right|>\varepsilon ; \\
& =\partial_{12}^{2} R_{p}\left(x_{1}, x_{2}\right), & & \left|x_{2}-x_{1}\right| \leq \varepsilon ;
\end{aligned}
$$

where $\partial_{i}$ denotes partial differentiation with respect to the $i$ th argument. Then, instead of (2.31a), in [1], we have

$$
\begin{aligned}
\left\langle g_{1}\left(x_{1}\right) g_{1}\left(x_{2}\right)\right\rangle= & R_{f}\left(x_{1}, x_{2}\right)+R_{q}\left(x_{1}, x_{2}\right) y_{0}\left(x_{1}\right) y_{0}\left(x_{2}\right) \\
& +R_{p}\left(x_{1}, x_{2}\right) y_{0}^{\prime \prime}\left(x_{1}\right) y_{0}^{\prime \prime}\left(x_{2}\right)+\partial_{1} R_{p}\left(x_{1}, x_{2}\right) y_{0}^{\prime}\left(x_{1}\right) y_{0}^{\prime \prime}\left(x_{2}\right) \\
& +\partial_{2} R_{p}\left(x_{1}, x_{2}\right) y_{0}^{\prime \prime}\left(x_{1}\right) y_{0}^{\prime}\left(x_{2}\right) \\
& +\partial_{12}^{2} R_{p}\left(x_{1}, x_{2}\right) y_{0}^{\prime}\left(x_{1}\right) y_{0}^{\prime}\left(x_{2}\right), \quad\left|x_{2}-x_{1}\right| \leq \varepsilon .
\end{aligned}
$$

The quantity $A_{1}(x)$ is still given by

$$
A_{1}(x)=2 \int_{0}^{1} G^{2}(x, z)\left\langle g_{1}^{2}(z)\right\rangle d z,
$$

but now

$$
\begin{aligned}
\left\langle g_{1}^{2}(z)\right\rangle= & R_{f}(z, z)+R_{q}(z, z) y_{0}^{2}(z)+R_{p}(z, z) y_{0}^{\prime \prime 2}(z) \\
& +\left[\partial_{1} R_{p}(z, z)+\partial_{2} R_{p}(z, z)\right] y_{0}^{\prime}(z) y_{0}^{\prime \prime}(z) \\
& +\partial_{12}^{2} R_{p}(z, z) y_{0}^{\prime 2}(z) .
\end{aligned}
$$

In a similar way

$$
A_{2}(x)=-\frac{1}{2}\left[G^{2}(x, 0)\left\langle g_{1}^{2}(0)\right\rangle+G^{2}(x, 1)\left\langle g_{1}^{2}(1)\right\rangle\right]
$$

where $\left\langle g_{1}^{2}(0)\right\rangle$ and $\left\langle g_{1}^{2}(1)\right\rangle$ are found from (A.9). Eqs. (3.30) for $A_{3}(x)$ and (4.41) for $A_{4}(x)$ remain valid, but $\left\langle g_{1}^{3}(z)\right\rangle$ and $\left\langle g_{1}^{4}(z)\right\rangle$ must be interpreted a little differently.

With these modifications in mind, the expressions (3.21) and (4.15) for $F_{\xi}(u)$ and (3.22) and (4.16) for $p_{\xi}(u)$ remain valid in this more general setting, as well as the results in Sec. 6. 
It is also possible to combine the results of Secs. 3 and 4 by calculating the term mentioned just above (4.5). This leads to the formula

$$
\begin{aligned}
p_{\xi}(u)=\phi(u)\{1 & +\left[\frac{A_{3} / A_{1}^{3 / 2}}{3 !} H_{3}(u)\right] \sqrt{ } \varepsilon \\
+ & {\left.\left[\frac{A_{2} / A_{1}}{2 !} H_{2}(u)+\frac{A_{4} / A_{1}^{2}}{4 !} H_{4}(u)+\frac{A_{3}^{2} / A_{1}^{3}}{72} H_{6}(u)\right] \varepsilon+O\left(\varepsilon^{3 / 2}\right)\right\} }
\end{aligned}
$$

with a corresponding result for $F_{\xi}(u)$. Of course, (A.11) reduces to (4.16) when $A_{3}=0$ and to (3.22) when only the $O\left(\varepsilon^{1 / 2}\right)$ term is retained.

\section{REFERENCES}

[1] William E. Boyce, Stochastic nonhomogeneous Sturm-Liouville problems, J. Franklin Inst. 282, 206-215 (1966)

[2] B. V. Gnedenko and A. N. Kolmogorov, Limit distributions for sums of independent random variables, Addison-Wesley Publishing Co., Inc., Cambridge, Massachusetts, 1954

[3] W. Purkert and J. vom Scheidt, Zur approximativen Lösung des Mittelungsproblems für die Eigenwerte stochastischer Differentialoperatoren, ZAMM 57, 515-525 (1977)

[4] W. Purkert and J. vom Scheidt, Randwertprobleme mit schwach korrelierten Prozessen als Koeffizienten, in Transactions of Eighth Prague Conference on Information Theory, Statistical Decision Functions, and Random Processes B, 107-118 (1978)

[5] W. Purkert and J. vom Scheidt, Ein Grenzverteilungssatz für stochastische Eigenwertprobleme, ZAMM 59, 611-623 (1979)

[6] W. Purkert and J. vom Scheidt, Stochastic eigenvalue problems for differential equations, Rep. Math. Phys. 15, 205-227 (1979)

[7] J. vom Scheidt and W. Purkert, Limit theorems for solutions of stochastic differential equation problems, Int. J. Math. and Math. Sci. 3, 113-149 (1980)

[8] G. E. Uhlenbeck and L. S. Ornstein, On the theory of Brownian motion, Phys. Rev. 36, 823-841 (1930) 